J3eA, Journal sur l'enseignement des sciences et technologies de l'information et des systèmes, Volume 4, Hors-Série 1, 5 (2005)

DOI : http://dx.doi.org/10.1051/bib-j3ea:2005605

(C) EDP Sciences, 2005

\title{
Actionneurs linéaires directs et indirects : performances limites
}

H. Ben Ahmed, B. Multon et P.E. Cavarec

Laboratoire SATIE (UMR 8029 CNRS)

Antenne de Bretagne, ENS Cachan

Campus de Ker Lann

F-35170 Bruz, France

benahmed@bretagne.ens-cachan.fr 


\title{
Actionneurs linéaires directs et indirects : performances limites
}

\author{
H. Ben Ahmed, B. Multon, P.E. Cavarec. \\ SATIE (UMR 8029 CNRS) - Antenne de Bretagne, ENS Cachan, Campus de Ker Lann, 35170 BRUZ ( ${ }^{1}$ )
}

Résumé- A partir d'une classification descriptive des actionneurs électromagnétiques et d'un formalisme simplifié de calcul de leurs performances, les auteurs tentent, dans cet article, de dégager et de comparer différentes solutions d'entraînement linéaire directs et indirects. En particulier, les performances volumiques et dynamiques limites sont analysées à partir des lois de similitude.

\section{INTRODUCTION}

Dans de nombreuses applications, il est nécessaire de disposer d'un mouvement de translation [1,3,4]. Comme l'illustrent les différents graphiques de la figure 1 [2], l'étendue des performances souhaitées, aussi bien en termes de puissances que de courses, est très élevée allant des applications à très forte accélération telles que les lanceurs électromagnétiques ( $\approx 1000 \mathrm{~g})$, à celles exigeant de très grandes vitesses de déplacement tels que les trains à propulsion magnétique $(\approx 100 \mathrm{~m} / \mathrm{s}$ ), en passant par les systèmes nécessitant un positionnement précis ( $\approx \mu \mathrm{m}$, industrie électronique), ou encore une poussée importante tels que l'usinage $(\approx 10 \mathrm{kN})$ ou les actionnements aéronautiques (voir figure 2) [5].

Les solutions adoptées sont généralement de deux types :

- entraînements indirects : ils restent très employés actuellement et nécessitent un organe de transmission et de conversion mécanique entre l'actionneur rotatif et sa charge. Dans le domaine aéronautique, les entraînements sont généralement de type hydraulique à puissance électrique, celleci étant prélevée sur le réacteur via un générateur électrique. Ces aspects seront sommairement abordés dans la dernière partie de cet article ;

- entraînement directs : ils visent à simplifier la chaîne de transmission par la suppression de l’organe intermédiaire.

Les solutions mettant en œuvre des dispositifs mécaniques et/ou hydrauliques intermédiaires de transformation (réducteur, vis écrou, pompe+vérin) permettent souvent l'obtention de performances massiques élevées. Cependant, ces intermédiaires introduisent deux types de limitations : plus faibles performances dynamiques (bande passante mécanique) et inconvénients tels que l'usure, le bruit ou la fiabilité.

Les solutions d'entraînement direct sont quant à elles limitées par des considérations physiques (magnétiques, thermiques, ...). Ces limitations interviennent différemment selon le type d'actionneur et selon son architecture.
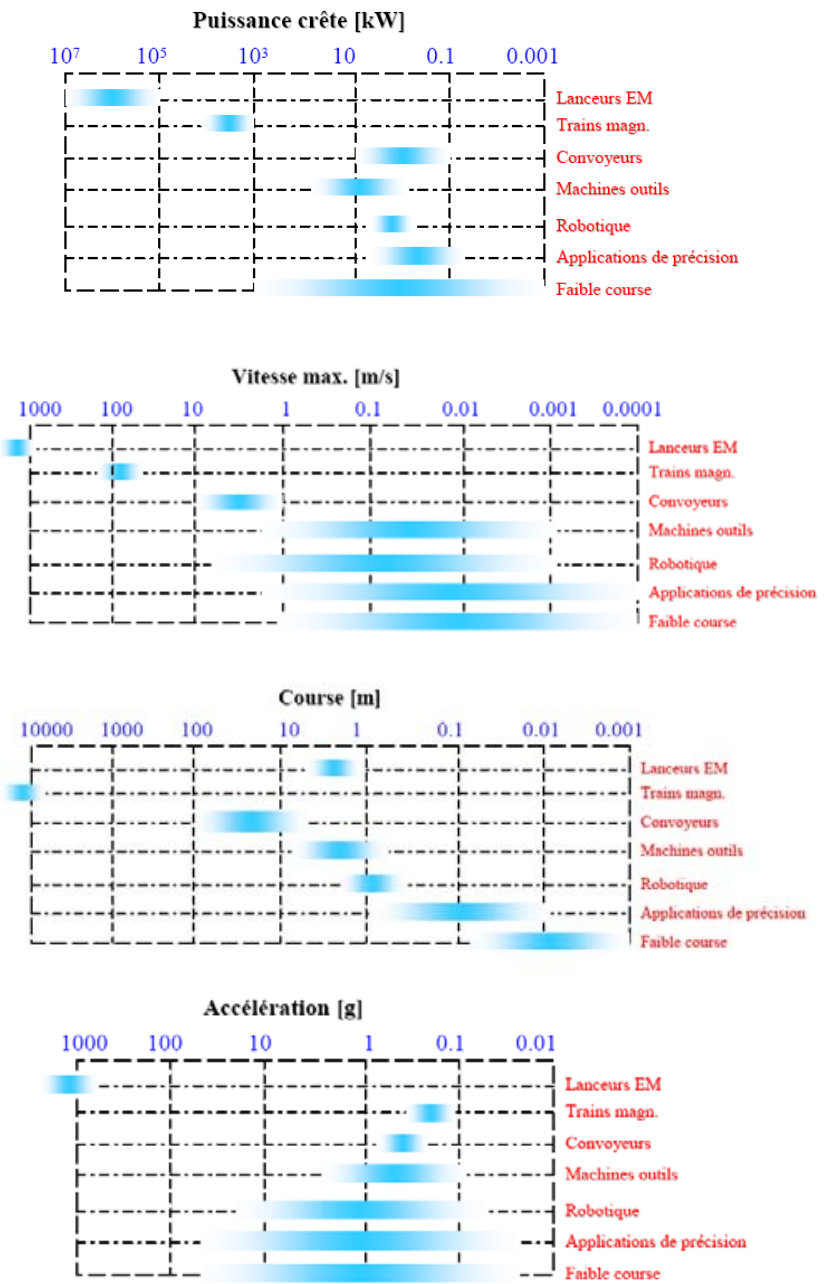

Fig. 1. Quelques ordres de grandeurs de performances attendues des actionneurs linéaires [2]

\begin{tabular}{|c|c|c|c|c|}
\hline \multirow[b]{2}{*}{ Systèmes } & \multirow[b]{2}{*}{ Désignation } & \multicolumn{3}{|c|}{ Caractéristiques } \\
\hline & & $\begin{array}{l}\text { Couple }[\mathrm{kN} . \mathrm{m}] \\
\text { Vitesse angulaire [\%/s] }\end{array}$ & & tement $\left[{ }^{\circ}\right]$ \\
\hline \multirow{3}{*}{ Cde de vol } & $\begin{array}{l}\text { Aileron } \\
\text { Dir. profondeur }\end{array}$ & $\begin{array}{l}1 \text { à } 20 \\
30 \text { à } 40 \text { max }\end{array}$ & 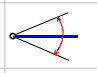 & 50 à 80 max \\
\hline & Bec / Volet & $\begin{array}{l}10 \text { à } 40 \\
2 \text { à } 5\end{array}$ & $30 \max$ & $\bar{\gamma}$ \\
\hline & $\begin{array}{l}\text { Plan horiz. } \\
\text { réglable }\end{array}$ & $\begin{array}{l}60 \text { à } 400 \\
0.5 \text { à } 1\end{array}$ & $\infty$ & $20 \max$ \\
\hline $\begin{array}{l}\text { Train } \\
\text { d'attérissage }\end{array}$ & $\begin{array}{l}\text { Avant } \\
\text { Principal }\end{array}$ & $\begin{array}{l}15 \text { à } 40 \\
15 \text { max }\end{array}$ & & $90 \max$ \\
\hline $\begin{array}{l}\text { Inverseur } \\
\text { de poussée }\end{array}$ & Porte & $\begin{array}{l}2.5 \text { à } 5 \\
40 \text { max }\end{array}$ & & $80 \max$ \\
\hline \multirow[b]{2}{*}{ Portes } & Cargo & $\begin{array}{l}0.5 \\
10\end{array}$ & & $120 \max$ \\
\hline & $\begin{array}{l}\text { Trappes train } \\
\text { d'attérissage }\end{array}$ & $\begin{array}{l}1.2 \text { à } 2.5 \\
60 \max \end{array}$ & & $90 \max$ \\
\hline
\end{tabular}

Fig. 2. Quelques applications potentielles dans l'aéronautique [5] 
Avant de nous intéresser plus précisément aux convertisseurs électromagnétiques (EM), examinons, plus généralement, les domaines de performances et d'applications potentielles des entraînements électromécaniques et électro-hydrauliques à travers quelques exemples d'actionneurs industriels ou au stade de la recherche et développement [6].

Considérons la puissance volumique développée par un actionneur donné. Celle-ci peut, d’une manière générale, s’écrire :

$$
\frac{\mathrm{P}}{\mathrm{V}_{\mathrm{a}}}=\left(\frac{\mathrm{F}}{\mathrm{S}_{\mathrm{a}}}\right)\left(\frac{\mathrm{v}}{\ell}\right)
$$

où $\mathrm{F}$ est la force moyenne motrice, $\mathrm{S}_{\mathrm{a}}$ la surface active (surface d'interface entre la partie fixe et la partie mobile, siège des échanges d'énergie et des forces), $\mathrm{v}$ la vitesse de déplacement de la partie mobile, $\ell$ est une dimension caractéristique de l'actionneur de volume actif $\mathrm{V}_{\mathrm{a}}$.

- Pour un actionneur cylindrique tournant (figure $3 a$ ), $\mathrm{F} / \mathrm{S}_{\mathrm{a}} \equiv \sigma_{\mathrm{t}}$ représente la pression tangentielle. $\mathrm{v} / \mathrm{\ell}=\mathrm{v} / \mathrm{R}_{\mathrm{e}}=\Omega$ est la vitesse de rotation. Ainsi, le rapport $\mathrm{P} / \mathrm{V}_{\mathrm{a}}$ correspond à la puissance ramenée au volume rotorique (cylindrée) ;

- Pour un actionneur linéaire dont le déplacement est normal à la surface active (figure $3 \mathrm{~b}$ ), $\mathrm{F} / \mathrm{S}_{\mathrm{a}} \equiv \sigma_{\mathrm{n}}$ représente la pression normale et $\ell$ correspond à la course ;

- Pour un actionneur linéaire dont le déplacement est tangent à la surface active (figure 3c), $\mathrm{F} / \mathrm{S}_{\mathrm{a}} \equiv \sigma_{\mathrm{t}}$ est la pression tangentielle, et $\ell \equiv \mathrm{h}$ est la hauteur de l'actionneur (diamètre du mover pour un actionneur tubulaire).

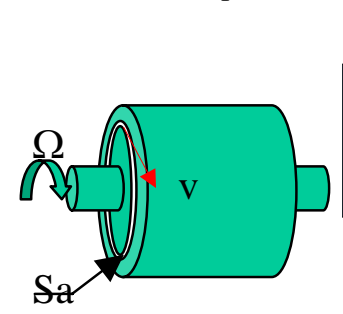

(a)

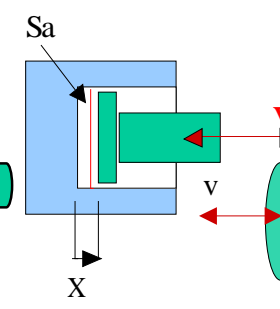

(b)

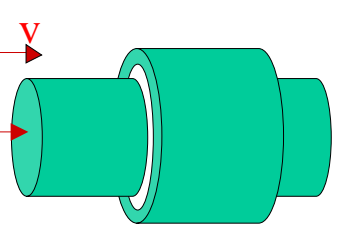

(c)
Fig. 3. Schémas d'actionneurs : (a) tournant cylindrique, (b) linéaire à déplacement normal, (c) linéaire à déplacement tangent

En utilisant l'expression (1), on peut ainsi qualitativement comparer les performances intrinsèques de différentes technologies d'entraînement dans le plan pression-vitesse relative. C'est ce qui est indiqué sur le diagramme de la figure 4 [6].

Ce diagramme montre ainsi, que pour les entraînements électromagnétiques $(\mathrm{EM})$, la puissance volumique générée est de l'ordre de $100 \mathrm{MW} / \mathrm{m}^{3}$, pour des vitesses relatives de $10^{2}$ à $10^{3} \mathrm{~s}^{-1}$. Les pressions magnétiques obtenues sont de l'ordre de quelques $\mathrm{N} / \mathrm{cm}^{2}$. Dans les entraînements hydrauliques, bien que bénéficiant des possibilités d'obtention de pressions élevées (100 à 1000 fois supérieures), la limitation en vitesse relative (quelques $\mathrm{s}^{-1}$ ) ne permet pas d'obtenir des puissances volumiques très importantes. Ces dernières sont en effet de même ordre de grandeur que celles des systèmes EM.

Signalons cependant que ce diagramme ne tient pas compte des effets d'échelle éventuels liés aux types de convertisseurs. C’est ce que nous allons examiner dans la suite de cet article en nous intéressant particulièrement aux actionneurs linéaires électromagnétiques directs et indirects après un bref rappel du principe de conversion et des topologies existantes.

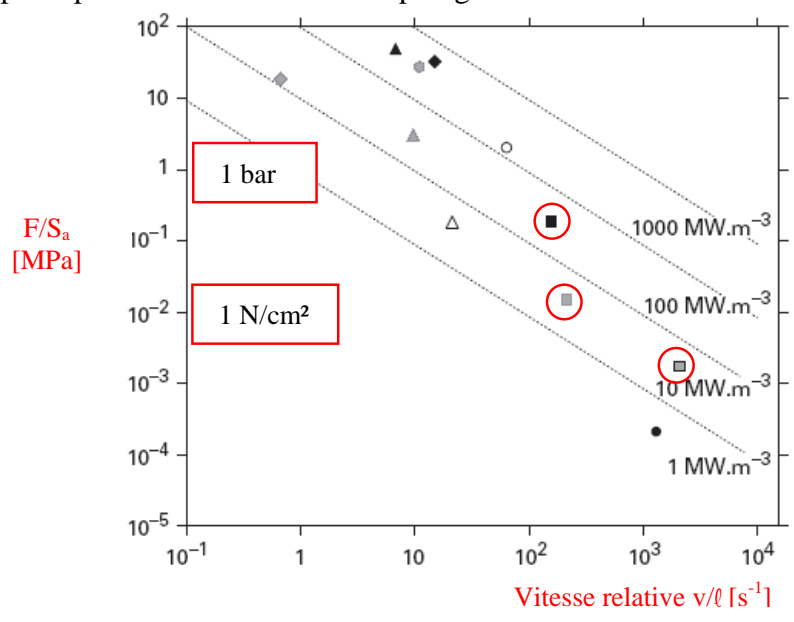

- Barreau piézoélectrique (PZT - multicouche)

- Barreau magnétostrictif (Terfénol D - transducteurs sonar)

- Actionneur hydraulique (gouverne aéronautique)

- Turboalternateur $1500 \mathrm{MW}$

Moteur de robotique à aimant permanent

므 Moteur d'électroménager (aspirateur)

Actionneurs EM

- Micromoteur à effet électrique

- Machine à vapeur (locomotive)

$\Delta$ Alliage à mémoire de forme

$\triangle$ Muscle biologique

$\Delta$ Transducteur piézoélectrique (stator à onde progressive)

Fig. 4. Ordres de grandeurs des efforts surfaciques et des vitesses relatives pour quelques technologies de convertisseurs électromécaniques [6]

\section{ENTRAINEMENTS EM DIRECTS}

\section{A. Principe}

Un convertisseur électromécanique d'énergie, indépendamment de son architecture, est un système dans lequel le transfert de puissance électrique-mécanique (en fonctionnement moteur) est réalisé à partir d'une conversion intermédiaire électromagnétique. D’une manière schématique (voir fig. 5), la magnétisation du système, en particulier celle de l'entrefer, est réalisée à travers deux types de sources magnétiques que l'on qualifie de puissance (bobinages permettant le transfert de puissance) et d'excitation (inducteur bobiné, réluctant, à aimants...). Dans le cas d'une excitation bobinée, la puissance absorbée se limite à ses propres pertes.

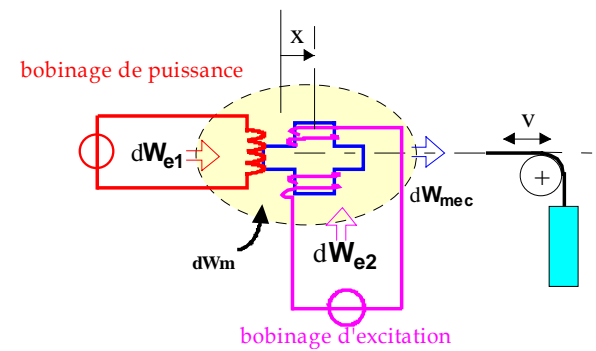

Fig. 5. Schéma de principe de conversion électromagnétique à deux sources magnétiques

La force générée dans un tel système est liée à la variation de son énergie magnétique par rapport au déplacement de sa partie mobile. Ainsi, pour un déplacement $\mathrm{x}_{\mathrm{j}}$ (rotation ou translation), la force généralisée (force ou couple) correspondante peut s’écrire [6, 7] : $F_{j}\left(x_{j}\right)=\frac{\partial W_{m}}{\partial x_{j}}$

La valeur moyenne de la force magnétique générée est donc égale au rapport de l'énergie $\Delta \mathrm{W}_{\mathrm{m}}$ convertie sur un cycle et la période mécanique de conversion $2 \tau$ : 
$\overline{\mathrm{F}}=\frac{\Delta \mathrm{W}_{\mathrm{m}}}{2 \tau}$

Un exemple de cycles de conversion idéalisés, représentés dans le plan flux/ampères-tours (force magnétomotrice), obtenu pour un actionneur synchrone classique non saturé dont la source de puissance est alimentée en courant sinusoïdal ou en créneaux est donné à la figure suivante.

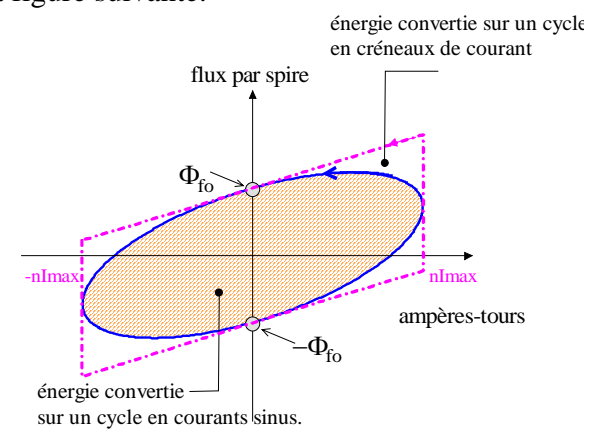

Fig. 6. Exemples de cycles de conversion électromécanique

Pour un actionneur comportant $\mathrm{N}_{\mathrm{b}}$ bobines au total $\left(\mathrm{N}_{\mathrm{b}}=2 \mathrm{mpq}\right.$ dans le cas classique d'une machine $2 \mathrm{p}$ pôles, $\mathrm{q}$ phases et $\mathrm{m}$ encoches/pôle/phase), la force moyenne générée s’écrit dans ce cas simplifié sous la forme :

$\overline{\mathrm{F}}=\mathrm{N}_{\mathrm{b}} \mathrm{k}_{\mathrm{imoy}} \frac{\pi \cdot \Phi_{\mathrm{fo}} \cdot \mathrm{nI}_{\max }}{2 \tau}$

où $\Phi_{\text {fo }}$ est le flux d'excitation (inducteur) embrassé par une spire du bobinage de puissance et $\mathrm{nI}_{\max }$ est la valeur crête de la force magnéto-motrice (ampères-tours) par bobine. $\mathrm{k}_{\text {imoy }}$ est un coefficient relatif à la forme d'onde du courant d'alimentation :

$\mathrm{k}_{\text {imoy }}= \begin{cases}1 & : \text { courants et flux inducteur sinusoïdaux } \\ 4 / \pi & : \text { courants créneaux et forme quelconque du flux } \\ \text { inducteur }\end{cases}$

On note :

$\overline{\mathrm{B}_{\mathrm{f}}}(\mathrm{x})=\frac{\Phi(\mathrm{x})}{\mathrm{S}_{\mathrm{p}}}=\overline{\mathrm{B}_{\mathrm{f} 0}} \cdot \mathcal{R}(\mathrm{x})$ : l'induction moyenne sous un pôle statorique de section $S_{p} \cdot \overline{B_{f 0}}$ est l'induction moyenne maximale et $\mathcal{R}(\mathrm{x})$ est une fonction de répartition (x est la position du mover).

L'expression (3) s'écrit alors :

$\overline{\mathrm{F}}=\mathrm{N}_{\mathrm{b}} \mathrm{S}_{\mathrm{p}} \mathrm{k}_{\mathrm{imoy}} \frac{\overline{\mathrm{B}_{\mathrm{f0}}} \cdot \mathrm{nI} \max }{2 \tau}$

\section{B. Classification}

Il est bien difficile et même hasardeux de réaliser une classification exhaustive des convertisseurs électromécaniques d'énergie. Cependant, à partir de quelques critères simples fonctionnels et structurels définis suite à une analyse des nombreuses structures existantes, nous avons pu établir une sorte de nomenclature descriptive des actionneurs qui se veut être aussi générique que possible [8, 20, 22].

\section{1) Source magnétique d'excitation}

La source de puissance étant nécessairement de type courant produit (alimentation), celle d'excitation en revanche peut être générée suivant différents procédés. En particulier, on peut distinguer celle produite par une alimentation (cas classique, bobinage d'excitation accessible) ou par une aimantation rigide (excitation par AP) et celle induite par la source de puissance (cas des actionneurs à induction par exemple). Ainsi, les principaux modes de génération de l'excitation peuvent être résumés dans le tableau 1 [9]. Sur les figures suivantes, sont donnés trois exemples d'actionneurs linéaires obéissant à cette première classification.

\begin{tabular}{|c|c|c|c|}
\hline \multicolumn{2}{|c|}{ Source d'excitation } & Non-dissipative & dissipative \\
\hline \multirow[t]{2}{*}{ Produite } & Aimantation & MS et MCC à AP & $* * *$ \\
\hline & Courant & $\begin{array}{l}\text { MS bobinée } \\
\text { (supra) }\end{array}$ & $\begin{array}{l}\text { MCC et MS } \\
\text { bobinées et } \\
\text { généralisée }\end{array}$ \\
\hline \multirow[t]{2}{*}{ Induite } & Aimantation & MRV pure & $\begin{array}{l}\text { Machine } \\
\text { hystérésis }\end{array}$ \\
\hline & Courant & $* * *$ & MAS à rotor en CC \\
\hline
\end{tabular}

TABLEAU 1. CRITERES FONCTIONNELS [9]

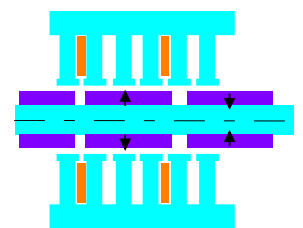

(a)

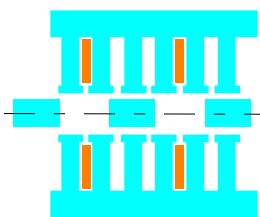

(b)

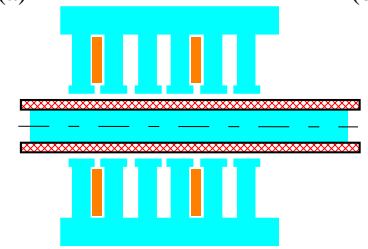

(c)

Fig. 7. Exemples de sources d'excitation : (a) : à aimants permanents, (b) : à réluctance variable pure, (c) : à courants induits

\section{2) Alimentation/mouvement}

Concernant les actionneurs linéaires, on peut de plus distinguer le type d'alimentation. Il s'agit ici de distinguer le pas de conversion $\tau$ lié à la fréquence d'alimentation du bobinage de puissance et à la course totale du mobile $\mathrm{X}$.

a) Actionneurs mono-pas

Dans cette première catégorie, les deux grandeurs caractéristiques définies précédemment sont très proches $(\tau / \mathrm{X} \approx 1)$. On trouve ici les actionneurs de type électroaimant ou encore haut-parleur.

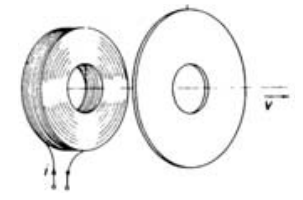

(a)
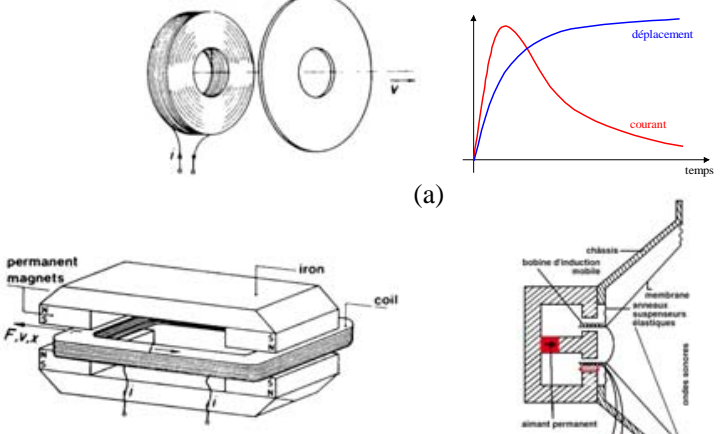

(b)
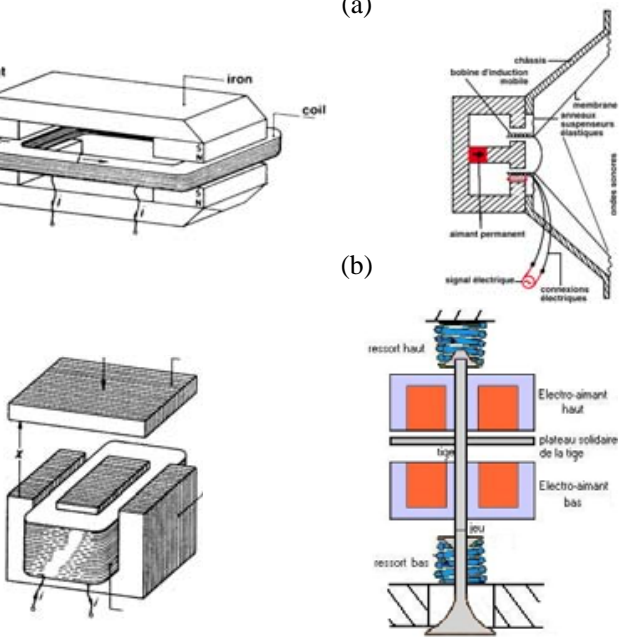

(c)

Fig. 8. Exemples d'actionneurs mono-pas : à courants induits (a), à bobine mobile (b) et électroaimant à réluctance variable (c). 
b)

\section{Actionneur multi-pas}

Dans cette catégorie, il y a dissociation totale entre la fréquence d'alimentation et la course $(\tau / \mathrm{X}<<1)$. On trouve ici la grande majorité des actionneurs électromagnétiques tournants ou linéaires. Dans la suite de l'étude, on s'intéressera uniquement aux actionneurs multi-pas.

\section{3) Couplages électromagnétiques (synchrones)}

Concernant le bobinage de puissance, celui-ci peut être globalement de deux types : le bobinage réparti (cas classique des actionneurs à champs tournants ou glissant dans lequel les différentes phases sont entrelacées) et le bobinage centralisé dentaire ou global. Dans ces deux derniers cas, les phases sont juxtaposées (couplages magnétiques inter-phases faibles), voire indépendantes (structures multistacks, couplages magnétiques totalement négligeables).

Le type d'interaction des deux sources de puissance et d'excitation générateur d'une force à valeur moyenne non nulle définit le couplage électromagnétique.

A partir du type de bobinage de puissance et des valeurs typiques du rapport $\tau / \tau_{\mathrm{p}}$ ( $\tau$ est lié à la fréquence fondamentale de conversion alors que $\tau_{\mathrm{p}}$ est lié à la dimension caractéristique du bobinage de puissance), on peut définir principalement 4 classes de machines élémentaires comme indiqué dans le tableau 2. En particulier, on distingue celles dont la géométrie du bobinage de puissance est associée à celle de la fréquence de conversion ( $\tau / \tau_{\mathrm{p}} \approx 1$, couplage dit polaire) et celles où ces deux grandeurs sont totalement dissociées $\left(\tau / \tau_{\mathrm{p}}<<1\right.$, couplage dit fractionné).

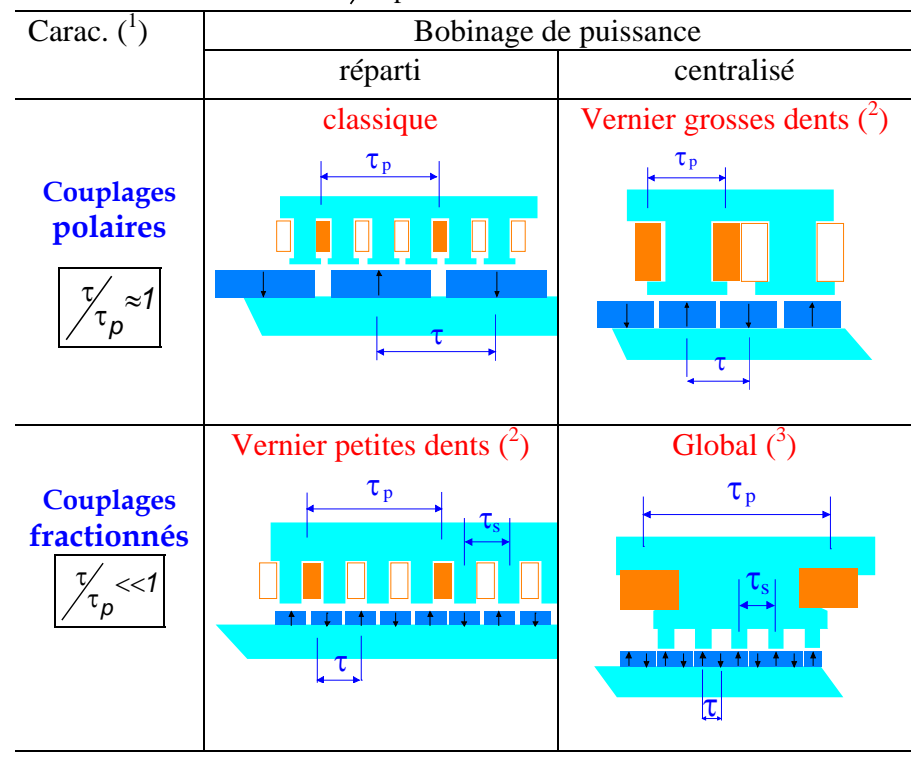

TABLEAU 2. COUPLAGES ELECTROMAGETIQUES [TI_PEC]

$\left(^{1}\right)$ Dans le cas classique $\tau_{p}$ traduit l'ouverture polaire.

$\left({ }^{2}\right)$ Dans ces deux types d'actionneurs Vernier, il existe une condition géométrique de bon fonctionnement : $\left|N_{a}-N_{s}\right|=p$ où $N_{a}$ et $N_{r}$ représentent respectivement le nombre de paires d'aimants rotor, et le nombre de dents stator, p étant le nombre de paires de pôles du bobinage statorique [10]. Cette condition s'écrit pour les actionneurs linéaires sous la forme: $\left|\frac{\tau_{\mathrm{p}}}{\tau}-2 \frac{{ }_{\mathrm{p}}}{\tau_{\mathrm{s}}}\right|=1$.

$\left({ }^{3}\right)$ Dans le cas des actionneurs à bobinage global, l'effet Vernier n'est pas fondamental (dans ce cas $\tau=\tau_{\mathrm{s}}$ ), cependant il peut être utilisé afin de minimiser les ondulations de force de détente ou de réluctance [11].

\section{4) Critères structurels}

Aux aspects mentionnés précédemment s’ajoutent les critères structurels de l'actionneur linéaire. Parmi ceux-ci, on peut noter :

a) Nombre de degrés de liberté

- $\quad$ Unique (cas classique)

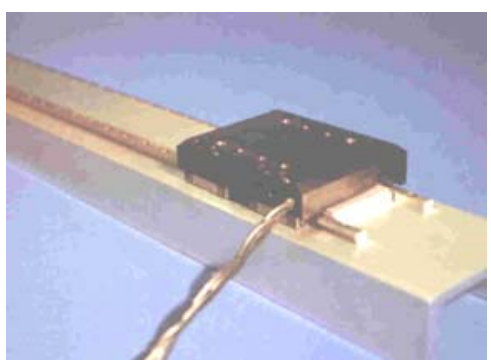

Fig. 9. Exemples d'actionneur à un degrés de liberté (translation suivant un seul axe) (ETEL)

- Multiple (mouvements combinés) : mouvement translation/rotation ou encore mouvement plan (actionneurs dits plans ou « planar actutators »).

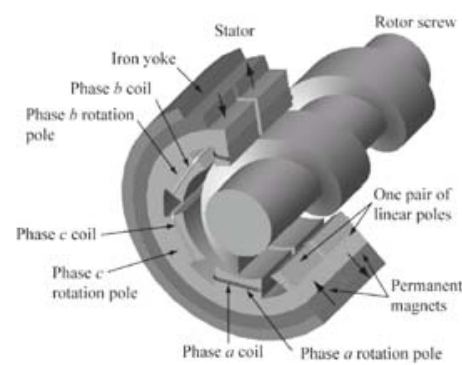

(a) [12]
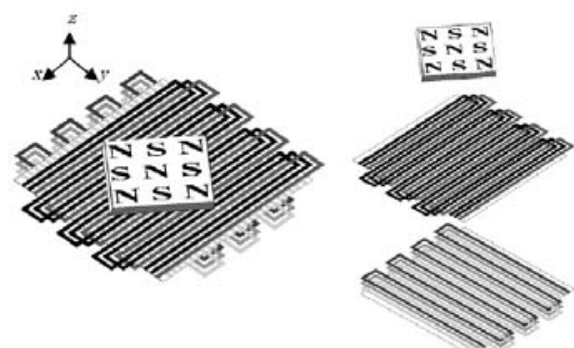

(b) [13]

Fig. 10. Exemples d'actionneurs à plusieurs degrés de liberté : rotation+translation (a), actionneur plan (b).

\section{b) Surface d'entrefer}

On peut distinguer des surfaces d'entrefer cylindriques (actionneurs tubulaire) ou planes (voir figure 11). La partie mobile ( "mover ») peut être entourée ou entourante. Enfin, dans le cas d'une surface plane, l'actionneur peut être à une seule ou encore à deux surfaces d'entrefer (structure dite en « $U$ »). Dans le premier cas, il subsiste des composantes d'effort, normales à la surface d'entrefer, non négligeables pouvant atteindre des valeurs 100 fois supérieures à celles des composantes tangentielles utiles. Ces efforts, devant être supportés par le système de guidage, rendent ce dernier délicat et peuvent générer des pertes par frottements importantes. A l'inverse, dans le cas des structures en $U$ ou tubulaires, la composante résultante des efforts normaux est partiellement compensée par le centrage. Néanmoins, l'équilibre obtenu est instable et il subsiste toujours un effort de décentrement ou de collage de la partie mobile sur la partie fixe. En effet, il est difficile d'avoir des positionnements parfaits assurant un centrage idéal des parties mécaniques. 
Signalons également que le nombre de surfaces d'entrefer peut être supérieur à deux, dans lequel cas, nous parlerons d'actionneurs polyentrefers. De telles architectures sont basées sur le fractionnement de la zone active en une multitude de zones élémentaires magnétisées par un bobinage unique (par phase) de type global. Cette disposition, bien que complexe mécaniquement, permet d'accroître sensiblement les forces volumiques par rapport aux structures monoentrefer [16]. Sur les figures 11e et 11f sont représentés deux exemples d'actionneur polyentrefers à aimants permanents, à bobinage global (couplage fractionné) et à champ longitudinal.

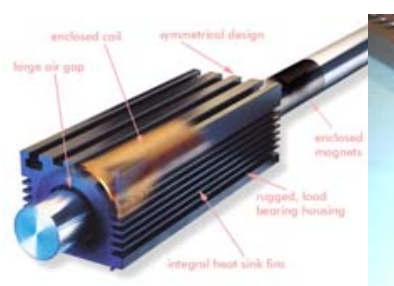

(a) cylindrique entourant (Lineardrives)

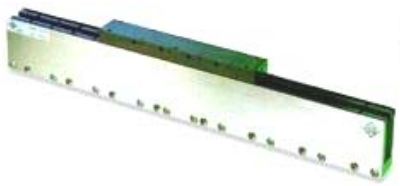

(c) plan bi-surfaces (Aerotech)

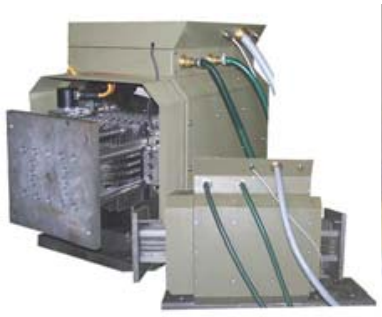

(e)

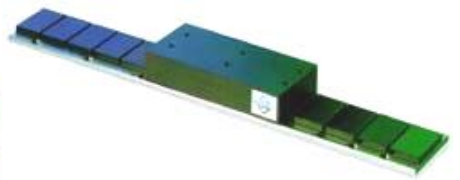

(d) plan mono-surface (Aerotech)

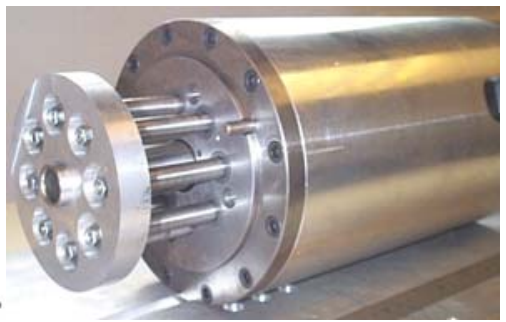

(f) (e), (f) : Exemples d'actionneurs linéaires polyentrefers (SATIE) (e) : architectrure multi-plaques [24], (f) : architecture multi-tiges [11].

Fig. 11 Exemples d'architectures selon la disposition des surfaces d'entrefer.

\section{c) Rail (excitation)}

Dans les actionneurs linéaires, la partie définissant la course est appelée rail. Ce dernier correspond souvent à la source d'excitation. Il peut être mobile ou fixe. A partir de la composition électrique et magnétique du rail, on peut définir des structures :

à rail actif (cas classique où le rail comporte des aimants permanents ou des matériaux électriques conducteurs) ;

à rail passif [14].

Dans le premier cas (figure 12a), la course et donc la longueur du rail influe notablement sur le coût global de l'actionneur. A l'inverse, un rail passif (figure 12b) est tout indiqué dans les applications à très grande course.

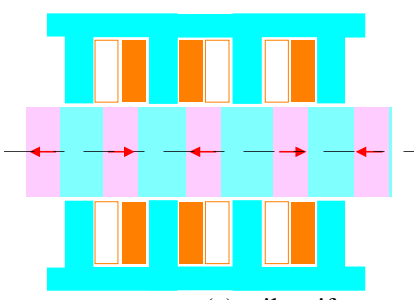

(a) rail actif

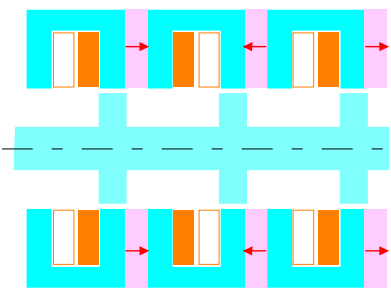

(b) rail passif
Fig. 12. Types de rail (schéma monophasé)
Les sources de puissance ou d'excitation peuvent êtres soit insérées dans un circuit magnétique comportant ainsi des encoches, soit mis dans l'entrefer sans intermédiaire magnétique soit enfin déportées.

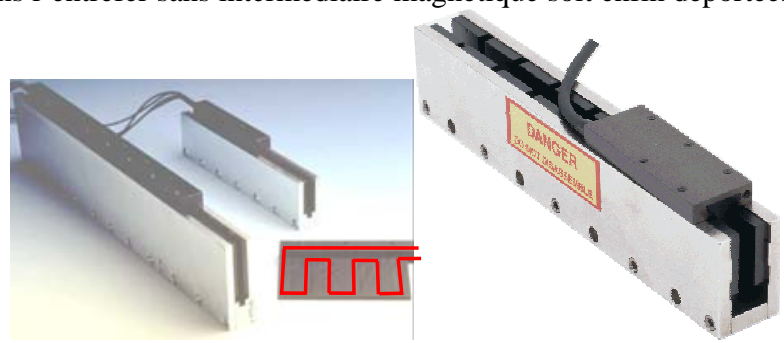

(a) bobinage dans l'entrefer de type réparti (Etel et Trilogy)

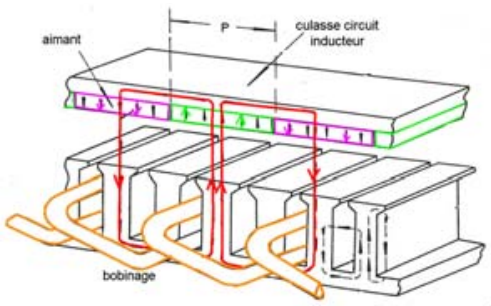

(b) bobinage dans les encoches de type réparti

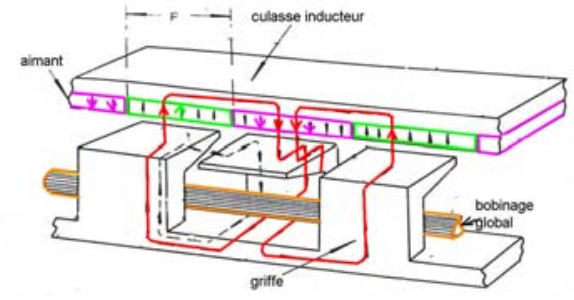

(c) bobinage déporté de type global

Fig. 13. Localisation de la source

Dans le premier cas (figure 13a) la source de puissance est située dans l'entrefer (pas d'encoche ni fer). Le bobinage est souvent autoporté, le cuivre se trouvant noyé dans une résine rigide. Cette disposition permet une grande facilité d'intégration mécanique et des constantes de temps électriques faibles. En revanche, la densité massique d'effort et la capacité thermique sont réduites.

Le deuxième cas (figure 13b) correspond aux actionneurs classiques. Cette disposition permet un bon refroidissement du bobinage et un entrefer magnétique faible et contrôlé. Ceci confère donc à ces structures des performances massiques relativement intéressantes.

Dans le dernier cas (figure 13c), le bobinage est éloigné (déporté) de la surface d'entrefer. La canalisation du flux généré s'effectue à travers un circuit magnétique de forme adéquate. De plus, dans cette configuration, le bobinage est généralement de type global. Sa réalisation (in situ ou préfabriqué) est aisée et on peut réaliser des coefficients de remplissage relativement élevés $(>0.6)$. Ces dispositions se prêtent mieux à des structures cylindriques que linéaires.

Notons enfin que dans le cas d'une excitation par aimants permanents, les dispositions avec circuit magnétique (à encoches ou déporté) permettent notamment la concentration de flux (cas des figures $12 \mathrm{a}$ et $12 \mathrm{~b})$.

e) Trajet du flux par rapport à la surface d'entrefer Le long de la surface d'entrefer, le flux inducteur peut être globalement normal à celle-ci (cas classique, figure 14a) ou longitudinal (figure 14b). Cette dernière configuration permet en 
particulier de concevoir des structures linéaires à bobinage global solénoïdal à bas coût (monoentrefer, figures 11b et 14b) ou à hautes performances volumiques (polyentrefer, figure 11e et 11f).

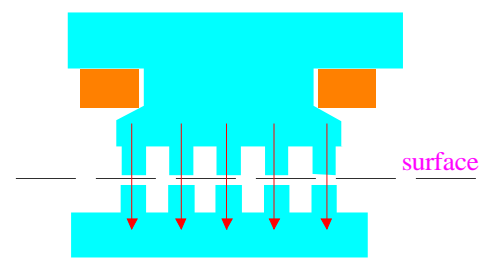

(a) flux normal (photo SATIE)
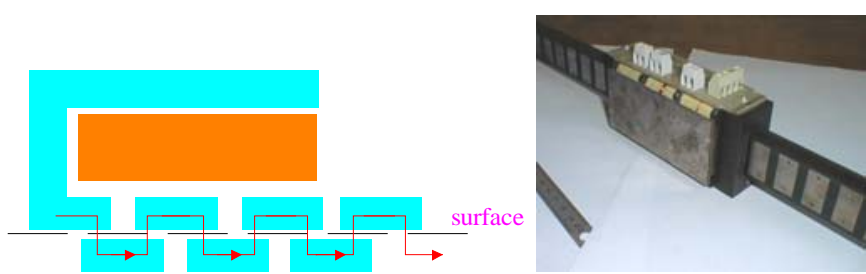

(b) flux longitudinal (photo SATIE)

Fig. 14. Trajet du flux d'entrefer

\section{Performances - Analyse aux lois de similitude}

Le calcul précis et rigoureux des performances des actionneurs électromagnétiques nécessite, pour chaque architecture, un travail de modélisation et d'optimisation lourd et complexe. Bien que précise, cette démarche conduit à une analyse difficile et parfois confuse des résultats obtenus. S'agissant dans notre cas d'une analyse comparative des performances des actionneurs électromagnétiques, notre choix s'est porté sur une modélisation simplifiée analytique. Cette dernière permet rapidement, à travers une analyse aux lois de similitude, d'évaluer les performances comparées et de dégager quelques règles simples de conception et de pré-dimensionnement.

\section{1) Système d'excitation}

L'induction d'excitation peut être obtenue soit par aimants permanents soit par courant d'excitation. Comparons tout d'abord ces deux modes d'excitation.
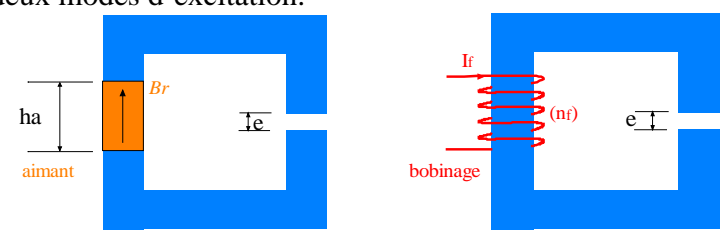

Fig. 15. Principes des systèmes d'excitation bobiné ou à aimant

En première approximation, on peut écrire :

$\mathrm{B}_{\mathrm{e}} \cong \mathrm{B}_{\mathrm{r}} \cdot \frac{1}{1+\mathrm{e} / \mathrm{h}_{\mathrm{a}}} \quad$ : excitation par aimants permanents

$\mathrm{B}_{\mathrm{e}} \cong \mu_{0} \frac{\mathrm{n}_{\mathrm{f}} \mathrm{I}_{\mathrm{f}}}{\mathrm{e}} \quad$ : excitation par bobine

où $\mathrm{n}_{\mathrm{f}} \mathrm{I}_{\mathrm{f}}=\mathrm{k}_{\mathrm{r}} \cdot \mathrm{s}_{\mathrm{b}} \cdot \delta$, avec $\mathrm{k}_{\mathrm{r}}$ coefficient de remplissage de cuivre, $\mathrm{s}_{\mathrm{b}}$ section bobinable et $\delta$ la densité de courant efficace $\left(^{2}\right.$ ).

En ne considérant que les pertes Joule dans l'évaluation des échauffements (dimensionnement en force quasi-statique), et en supposant que celui-ci s'effectue principalement par convection, nous pouvons alors écrire en régime thermique permanent :

\footnotetext{
${ }^{2}$ En annexe est donnée une liste des principales notations utilisées.
}

$\rho \delta^{2} \mathrm{v}_{\mathrm{cu}}=\alpha \Delta \theta_{\text {max }} \mathrm{S}_{\mathrm{th}}$

où $\mathrm{v}_{\mathrm{cu}}$ est le volume de cuivre, $\mathrm{S}_{\mathrm{th}}$ est la surface d'échange thermique et $\alpha$ le coefficient d'échange en $\mathrm{W} \cdot \mathrm{m}^{-2} \cdot \mathrm{K}^{-1}$.

Ce qui donne :

$$
\begin{aligned}
& \delta=\sqrt{\frac{\alpha \cdot \Delta \theta_{\text {max }}}{\rho} \cdot \sqrt{\frac{\mathrm{S}_{\text {th }}}{\mathrm{v}_{\mathrm{cu}}}}} \text { et } \quad \delta=\frac{\mathrm{n}_{\mathrm{f}} \mathrm{I}_{\mathrm{f}}}{\mathrm{s}_{\mathrm{b}} \cdot \mathrm{k}_{\mathrm{r}}} \\
& \mathrm{n}_{\mathrm{f}} \mathrm{I}_{\mathrm{f}}=\sqrt{\frac{\alpha \Delta \theta_{\max }}{\rho}} \cdot \sqrt{\frac{\mathrm{S}_{\mathrm{th}}}{\mathrm{v}_{\mathrm{cu}}}} \cdot\left(\mathrm{s}_{\mathrm{b}} \mathrm{k}_{\mathrm{r}}\right)
\end{aligned}
$$

Si l’on applique une homothétie sur toutes les dimensions excepté l'entrefer, il vient alors :

$\delta \propto \ell^{-0,5}, \mathrm{n}_{\mathrm{f}} \mathrm{I}_{\mathrm{f}} \propto \ell^{1,5}$ et $\left(\mathrm{B}_{\mathrm{e}}\right) \propto \frac{(\ell)^{1,5}}{\mathrm{e}}$

où $\ell$ est la dimension caractéristique du dispositif

Ainsi, l'induction d'excitation par aimants permanents semble indépendante des dimensions du système (pourvu que $\mathrm{e} / \mathrm{h}_{\mathrm{a}}$ reste faible), alors que celle obtenue par bobinage en dépend. Ceci traduit l'efficacité d'une excitation par aimants pour les faibles dimensions de pôles (figure 16).

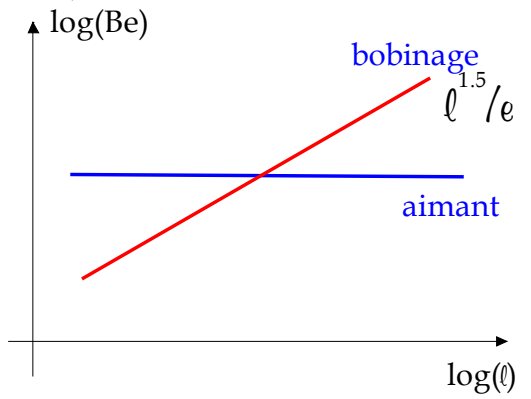

Fig. 16. Comparaison des deux systèmes d'excitation

\section{2) Poussées}

A la lumière de la précédente analyse, nous considérons désormais uniquement le cas des actionneurs à excitation par aimants permanents (aimantation alternée par exemple) de forme tubulaire. L’induction d'excitation sera considérée constante.

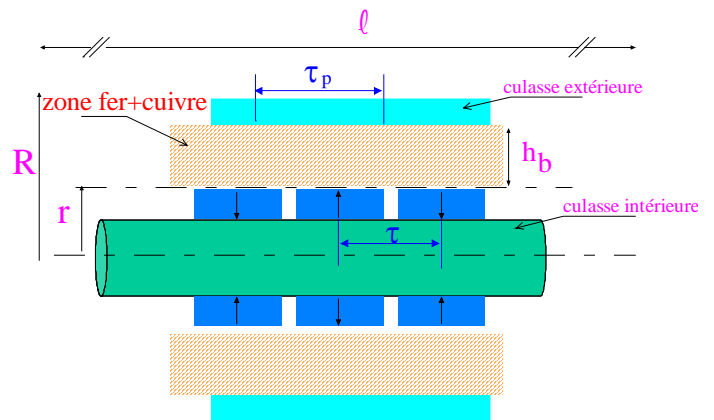

Fig. 17 Exemples d'actionneurs linéaires tubulaires à couplage polaire

\section{a) Formalisme :}

En appliquant la relation (7) à l'induit, nous obtenons :

$\mathrm{nI}_{\max }=\frac{1}{\mathrm{~N}_{\mathrm{b}}} \mathrm{k}_{\text {ieff }} \sqrt{\frac{\alpha \cdot \Delta \theta_{\max }}{\rho}} \cdot \sqrt{\frac{\mathrm{S}_{\mathrm{th}} \cdot \mathrm{S}_{\mathrm{b}}}{\ell_{\mathrm{b}}} \cdot \mathrm{k}_{\mathrm{r}}}$

où $\ell_{\mathrm{b}}$ est la longueur moyenne d'une spire et $\mathrm{k}_{\mathrm{ieff}}$ est donné par :

$\mathrm{k}_{\text {ieff }}=\frac{\mathrm{I}_{\max }}{\mathrm{I}}$

Pour une architecture tubulaire, nous pouvons écrire approximativement : 


$$
\begin{array}{ll}
\mathrm{S}_{\mathrm{th}} \approx 2 \pi \mathrm{R} \cdot \ell & : \text { surface d'échange thermique } \\
\mathrm{S}_{\mathrm{b}} \approx \mathrm{h}_{\mathrm{b}} \ell \mathrm{k}_{\mathrm{fc}} & : \text { section totale bobinable } \\
\ell_{\mathrm{b}} \approx 2 \pi\left(\mathrm{r}+\mathrm{h}_{\mathrm{b}} / 2\right) & : \text { longueur moyenne d'une spire } \\
\mathrm{S}_{\mathrm{p}} \approx 2 \pi \mathrm{r} \tau & : \text { section d'un pôle statorique } \\
\mathrm{R} \approx \mathrm{r}\left(1+\mathrm{h} / \mathrm{r}+\mathrm{e}_{\mathrm{c}} / \mathrm{r}\right) \approx \mathrm{r}(1+\mathrm{h} / \mathrm{r}) \text { (ép. culasse ext. } \mathrm{e}_{\mathrm{c}} \text { négligée) }
\end{array}
$$

$\mathrm{k}_{\mathrm{fc}}$ correspond à un taux de remplissage axial fer/cuivre dépendant en particulier du niveau de l'induction maximale dans les dentures statoriques.

$\mathrm{k}_{\mathrm{gr}}=\mathrm{k}_{\mathrm{r}} \mathrm{k}_{\mathrm{cf}}$ représente le coefficient global de remplissage de cuivre et $h_{b}$ est la hauteur de la fenêtre bobinable.

Ces approximations permettent d'écrire l'expression des ampèrestours sous une forme simple :

$\mathrm{nI}_{\max } \approx \frac{\ell}{\mathrm{N}_{\mathrm{b}}} \mathrm{k}_{\mathrm{ieff}} \sqrt{\frac{\alpha \cdot \Delta \theta_{\max }}{\rho}} \cdot \sqrt{\frac{h_{h}(1+h)}{\left(1+\frac{h}{2}\right)} \cdot \mathrm{k}_{\mathrm{gr}}}$

où $h=\frac{h_{b}}{r}$ : hauteur relative de la fenêtre bobinable.

En injectant cette expression dans l'équation (4), on obtient :

$$
\overline{\mathrm{F}}=\mathrm{k}_{\text {imoy }} \mathrm{k}_{\mathrm{ieff}} \sqrt{\frac{\alpha \cdot \Delta \theta_{\text {max }}}{\rho}} \overline{\mathrm{B}_{\mathrm{f} 0}} \cdot \ell \mathrm{r}^{\frac{3}{2}} \cdot \sqrt{\frac{h(1+h)}{\left(1+\frac{h}{2}\right)} \cdot \mathrm{k}_{\mathrm{gr}}}(\tau / \mathrm{p} / \tau)
$$

Le volume actif de l'actionneur vaut approximativement :

$\mathrm{V}_{\mathrm{a}}=\pi \mathrm{R}^{2} \cdot \ell \approx \pi \mathrm{r} \cdot(1+h)^{2} \cdot \ell$

Ce qui donne un effort volumique :

$\frac{\overline{\mathrm{F}}}{\mathrm{V}_{\mathrm{a}}} \approx \mathrm{k}_{\text {imoy }} \cdot \mathrm{k}_{\text {ieff }} \sqrt{\mathrm{k}_{\mathrm{gr}}}\left(\mathrm{A}_{\mathrm{L}} \cdot \delta\right)^{0,5} \overline{\mathrm{B}_{\mathrm{f} 0}} \cdot \mathscr{F}_{h} \cdot \mathrm{r}^{-0,5}\left(\tau_{\mathrm{p}} / \tau\right)$

où $\mathcal{F}_{h}=\sqrt{\frac{h}{(1+h)^{3}(1+h / 2)}}$

Cette quantité dont la variation est représentée à la figure 18, admet un optimum tel que :

$h_{\text {opt }}=0,4$ et $\mathscr{F}_{\text {opt }} \approx 0,35$

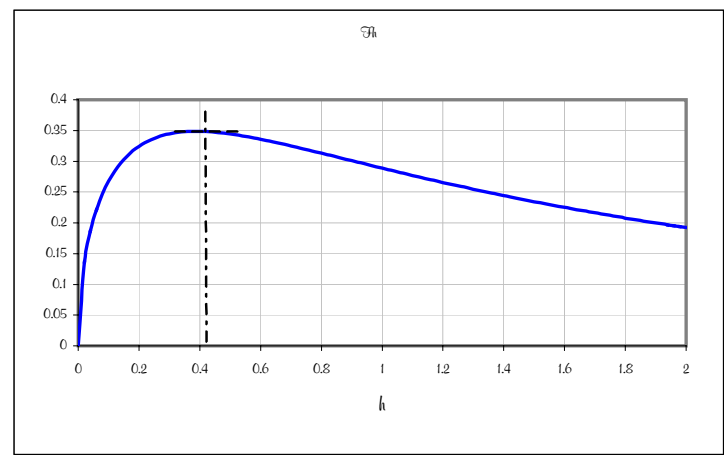

Fig. 18 Variation de $\mathcal{F}_{h}$ en fonction de $h$

Ainsi, la force et la force volumique peuvent finalement s'écrire sous la forme :

$\overline{\mathrm{F}} \approx \mathrm{K}_{\mathrm{f}}\left(\mathrm{A}_{\mathrm{L}} \cdot \delta\right)^{0,5} \overline{\mathrm{B}_{\mathrm{f} 0}}\left(\tau_{\mathrm{p}} / \tau\right) \ell \mathrm{r}^{\frac{3}{2}}$

$$
\frac{\overline{\mathrm{F}}}{\mathrm{V}_{\mathrm{a}}} \approx \mathrm{K}_{\mathrm{fv}}\left(\mathrm{A}_{\mathrm{L}} \cdot \delta\right)^{0,5} \overline{\mathrm{B}_{\mathrm{f} 0}}(\tau \mathrm{p} / \tau) \frac{1}{\sqrt{\mathrm{r}}} \text { avec } \mathrm{r} \propto \mathrm{R}
$$

où :

$$
\begin{aligned}
& \mathrm{K}_{\mathrm{f}} \approx \mathrm{k}_{\text {imoy }} \mathrm{k}_{\text {ieff }} \cdot \pi \sqrt{\sqrt{\left(\frac{h_{\text {opt }}\left(1+h_{\text {opt }}\right)}{\left.1+\frac{h_{\text {opt }}}{2}\right)} \cdot \mathrm{k}_{\mathrm{gr}}\right.} \approx 2,15 \cdot \mathrm{k}_{\text {imoy }} \mathrm{k}_{\mathrm{ieff}} \cdot \sqrt{\mathrm{k}_{\mathrm{gr}}}} \\
& \mathrm{K}_{\mathrm{fv}} \approx \mathrm{k}_{\text {imoy }} \cdot \mathrm{k}_{\text {ieff }} \sqrt{\mathrm{k}_{\mathrm{gr}} \cdot \mathcal{F}_{\mathrm{opt}}}
\end{aligned}
$$

Remarque :

On note $: \frac{\mathrm{N}_{\mathrm{b}} \mathrm{nI}}{\ell} \equiv \mathrm{A}_{\mathrm{L}}$ où $\mathrm{A}_{\mathrm{L}}$ représente la densité linéique efficace de courant. Nous retrouvons, comme dans les machines tournantes cylindriques la limitation thermique symbolisée par le produit $\left(\mathrm{A}_{\mathrm{L}} \cdot \delta\right)$ tel que :

$\left(\mathrm{A}_{\mathrm{L}} \cdot \delta\right)=\frac{\alpha \Delta \theta_{\max }}{\rho}\left(\frac{\mathrm{S}_{\mathrm{th}}}{\ell \cdot \ell_{\mathrm{b}}}\right) \approx \frac{\alpha \Delta \theta_{\max }}{\rho}$

A titre d'exemple, pour $\Delta \theta_{\max }=100 \mathrm{~K}$ et $\rho=2.410^{-8} \Omega \mathrm{m}$, nous obtenons $50 \mathbf{A}^{2} / \mathbf{m m}^{3}$ pour $\alpha=12 \mathrm{~W} / \mathrm{m}^{2} . \mathrm{K}$ (convection naturelle).

D'autre part, en injectant l'expression de $A_{L}$ dans celle de la force moyenne, on montre que :

$\overline{\mathrm{F}}=\left[\frac{\mathrm{k}_{\text {imoy }} \mathrm{k}_{\text {ieff }}}{2} \cdot \frac{\tau}{\tau} \mathrm{A}_{\mathrm{L}} \overline{\mathrm{B}_{\mathrm{f} 0}}\right] \cdot 2 \pi \mathrm{r}$

Le terme entre crochets correspond à la pression tangentielle magnétique moyenne (en $\mathrm{N} / \mathrm{m}^{2}$ ).

A partir de ce formalisme et principalement des relations (14) et (15), nous allons examiner les performances des deux couplages principaux précédemment définis.

b) $\quad$ Couplages polaires $\left(\tau_{\mathrm{p}}^{\mathrm{p}} / \tau \approx 1\right)$ :
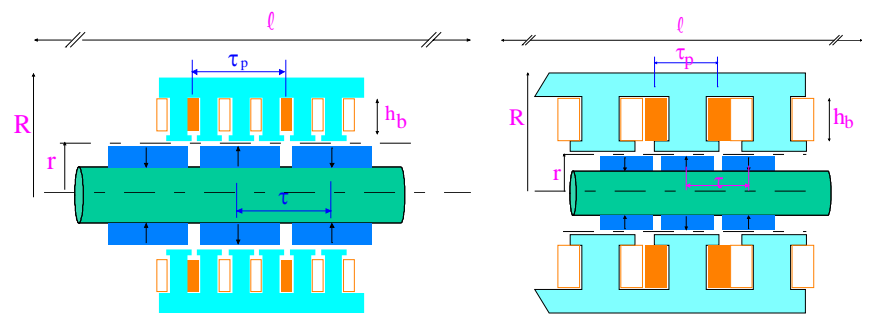

Fig. 19 Exemples d'actionneurs linéaires tubulaire à couplage polaire

Dans le cas des actionneurs à couplages polaires aussi bien classiques que «Vernier grosses dents», nous avons $\tau_{\mathrm{p}} / \tau \approx 1$. Ainsi :

$$
\begin{aligned}
& \overline{\mathrm{F}} \propto \ell \mathrm{r}^{\frac{3}{2}} \\
& \frac{\overline{\mathrm{F}}}{\mathrm{V}_{\mathrm{a}}} \propto \mathrm{r}^{-\frac{1}{2}} \text { avec } \mathrm{r} \propto \mathrm{R}
\end{aligned}
$$

Bien qu'approximative, ces deux relations mettent en évidence quelques résultats intéressants :

- la force volumique est indépendante de la longueur active $\ell$ de l'actionneur ;

- cette même force est également indépendante de la hauteur relative de la fenêtre bobinable : $h_{\text {opt }} \approx 0,4$; 
- la force volumique est inversement proportionnelle au rayon d'entrefer et donc au rayon extérieur ( $\mathrm{h}_{\mathrm{b}} / \mathrm{r}$ étant fixé). La valeur minimale de $\mathrm{r} \equiv \mathrm{r}_{\mathrm{opt}}$ est le résultat d'un compromis fonction de certaines considérations telles que :

- la hauteur minimale des aimants : en deçà d'une certaine valeur (dépendant de l'entrefer réalisable et du demi-pas polaire minimal $\tau_{\min }$ ), $\overline{\mathrm{B}_{\mathrm{f} 0}}$ décroît sensiblement. De plus, compte tenu des ampères-tours d'induit imposés, la diminution de la hauteur des aimants est également limitée par la désaimantation.

- l'épaisseur minimale de la culasse intérieure tributaire de l'induction de saturation du matériaux magnétique, de $\overline{\mathrm{B}_{\mathrm{f} 0}}$ et enfin de $\tau_{\min }$. Dans le cas des structures à aimants en surface, nous avons :

$$
\mathrm{r} \geq\left(\overline{\frac{\mathrm{B}_{\mathrm{f} 0}}{\mathrm{~B}_{\mathrm{m}}}}\right) \tau_{\mathrm{p}}+\mathrm{h}_{\mathrm{a}} \text { d'où : } \mathrm{r}>\left(\frac{\overline{\mathrm{B}_{\mathrm{f} 0}}}{\mathrm{~B}_{\mathrm{m}}}\right) \cdot \tau_{\mathrm{p}}
$$

où $\mathrm{B}_{\mathrm{m}}$ est l'induction maximale dans la culasse et $\mathrm{h}_{\mathrm{a}}$ est la hauteur des aimants.

Notons de plus que $\tau_{\min }$ est conditionné par son influence sur $\overline{\mathrm{B}_{\mathrm{f} 0}}$ et par les contraintes mécaniques de réalisation et de montage.

- Enfin, la force moyenne est proportionnelle à la longueur active $\ell$ et au rayon d'entrefer. L'augmentation de celle-ci en agissant sur $\ell$ plutôt que sur $r$ s'avers être plus efficace car dans le premier cas la force volumique est conservée.

En définitive, on peut conclure que la force volumique des actionneurs à couplages polaires $\left(\tau_{\mathrm{p}} / \tau \approx 1\right)$ est invariante vis à vis des principales dimensions et peut donc s'écrire :

$$
\frac{\overline{\mathrm{F}}}{\mathrm{V}_{\mathrm{a}}} \approx \mathrm{K}_{\mathrm{fv}}\left(\mathrm{A}_{\mathrm{L}} \cdot \delta\right)^{0,5} \cdot \overline{\mathrm{B}_{\mathrm{f} 0}} \cdot \frac{1}{\sqrt{\mathrm{r}_{\mathrm{opt}}}}=\left(\frac{\overline{\mathrm{F}}}{\mathrm{V}_{\mathrm{a}}}\right)_{\max } \approx \text { Constante }
$$

En guise d'application, pour $\left(\delta . \mathrm{A}_{\mathrm{L}}\right) \approx 50 \mathrm{~A}^{2} / \mathrm{mm}^{3}, \overline{\mathrm{B}_{\mathrm{f} 0}} \approx 0,7 \mathrm{~T}$, $\mathrm{k}_{\mathrm{gr}} \approx 0,25, \quad \mathcal{F}_{\mathrm{opt}} \approx 0,35$ et $\mathrm{r}_{\mathrm{opt}} \approx 10 \mathrm{~mm}$, on obtient $\mathrm{K}_{\mathrm{f}} \approx 1,5$, $\mathrm{k}_{\mathrm{fv}} \approx 0,25$ pour une alimentation en courants sinusoïdaux et $\mathrm{K}_{\mathrm{f}} \approx 1,4$, $\mathrm{k}_{\mathrm{fv}} \approx 0,22$ pour une alimentation en créneaux de courants. Ce qui donne respectivement $\mathbf{F} / \mathbf{V a} \approx \mathbf{4 0 0} \mathbf{N} / \mathbf{d m}^{\mathbf{3}}$ et $350 \mathrm{~N} / \mathrm{dm}^{3}\left({ }^{3}\right)$, valeur effectivement observée en pratique.

Signalons que la différence majeure entre les architectures classiques et Vernier grosses dents réside dans la réalisation du bobinage. Le bobinage centralisé permet d'obtenir souvent des coefficients de remplissage de cuivre meilleurs (à travers notamment sa compression [15]), de limiter la longueur des têtes de bobines (et donc le volume cuivre) pour les actionneurs à surface d'entrefer plane ou pour les actionneurs tournants et enfin de réaliser des pas polaires relativement faibles. Ces considérations ne modifient pas fondamentalement les résultats obtenus précédemment cependant cela se traduit par des performances volumiques légèrement plus faibles pour les actionneurs classiques comparativement aux actionneurs Vernier grosses dents. Ceci peut être quantifié à travers l'expression (19), en utilisant un coefficient $\mathrm{k}_{\mathrm{gr}}$ plus important et un pas minimal $\tau_{\min }$ et donc $\mathrm{r}_{\mathrm{opt}}$ plus faibles.

${ }^{3}$ Toutes les illustrations numériques ultérieures seront données par rapport à ces conditions.

\section{Exemple de calcul numérique :}

Pour valider l'approche développée précédemment, nous avons effectué un calcul de modélisation/optimisation sur un cas simple d'actionneur de type Vernier grosses dents (tableau 2) monophasé [16]. Il s'agissait, à volume donné, de maximiser la force moyenne compte tenu des limites de saturation (donc en régime non-linéaire), de désaimantation et d'échauffement. Le calcul des performances électromagnétiques a été réalisé à partir d’un code éléments finis non-linéaire 2D, les aspects thermiques ont été pris en compte suivant un modèle simplifié par résistances thermiques équivalentes et enfin, l'optimisation a été effectuée en utilisant une approche par algorithme génétique. Le schéma global de ce calcul est illustré par la figure 20. Afin de limiter le temps de calcul, on s'est restreint à la modélisation d'un motif élémentaire de l'actionneur donné à la figure 21 .

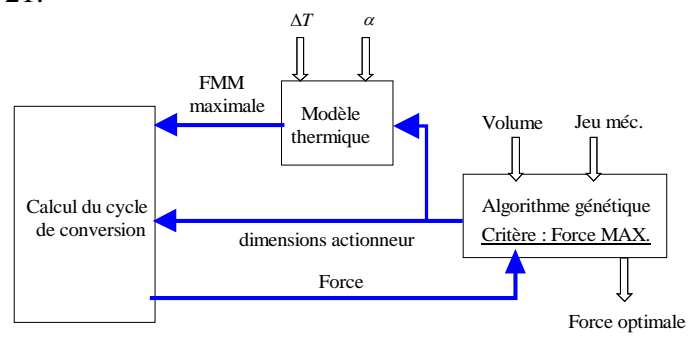

Fig. 20 Schéma global d’optimisation

La force volumique obtenue est donnée à la figure 22 où elle montre son invariance vis à vis du volume. L'algorithme d'optimisation sélectionnait toujours la forme la plus allongée possible (compte tenue du volume imposé) sans modifier les dimensions du motif élémentaire.

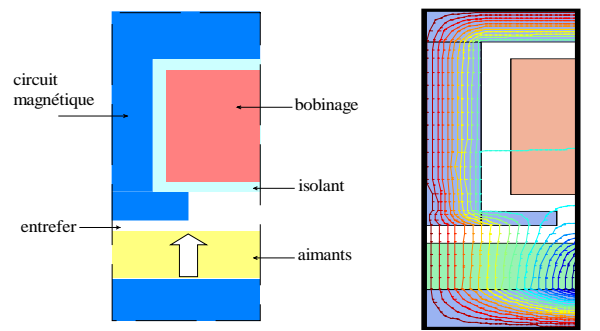

Fig. 21 Motif élémentaire et carte du champ inducteur [16]

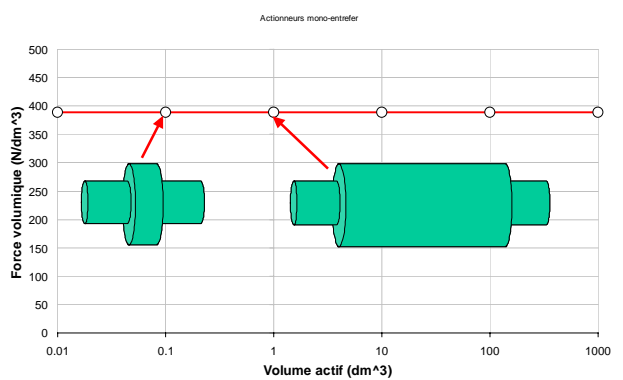

Fig. 22 Performances volumiques optimisées $\left(\mathrm{Br}=1 \mathrm{~T}, \alpha=10 \mathrm{~W} / \mathrm{m}^{2} . \mathrm{K}\right.$, $\Delta \theta \max =100 \mathrm{~K}, \mathrm{e}=1 \mathrm{~mm}, \mathrm{Bsat}=1.6 \mathrm{~T})[16]$

Nous venons de voir que pour maintenir l'effort volumique maximal, le rayon d'entrefer devra être maintenu constant. L'obtention d'un effort important passe donc nécessairement par l'allongement de l'actionneur.

Une telle solution n'est évidement pas viable en particulier pour les valeurs de force élevées (problèmes de tenue mécanique, encombrement limité en longueur, réalisation, flexion, guidage, ....). Pour pallier ces limitations, deux solutions s’offrent généralement au concepteur :

- la première consiste à augmenter le diamètre de l'actionneur proportionnellement à la longueur $r \propto \mathrm{R} \propto \ell$. Dans cette configuration, comme le montre les relations (16) et (17), la 
force augmente en $r^{\frac{5}{2}}$, et la force volumique se dégrade en $\mathrm{r}^{-\frac{1}{2}}$

- la seconde solution consiste à augmenter la force globale sans modifier la géométrie optimale de l'actionneur en disposant en "parallèle » de plusieurs actionneurs tubulaires optimisés (configuration multi-moteurs). Si la surface d'échange thermique de chaque actionneur est maintenue constante (en faisant circuler un fluide de refroidissement au sein de cet assemblage) alors la force volumique globale est approximativement conservée à sa valeur optimale, la force totale étant égale à la somme des forces générées par chaque actionneur. Cependant, cette solution accroît sensiblement la complexité mécanique du dispositif en particulier concernant le guidage, le refroidissement est plus délicat.

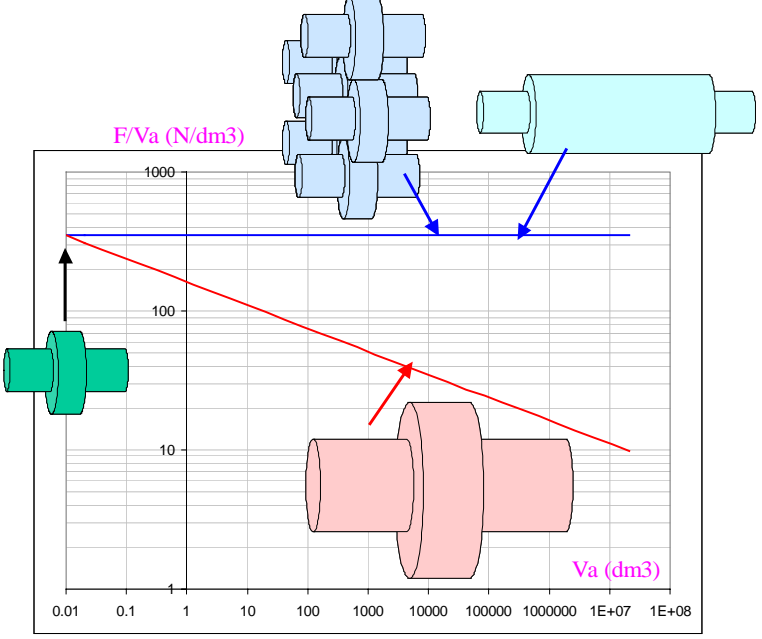

Fig. 23 Force volumique pour actionneur à couplage polaire : différentes dispositions

c) Couplages fractionnés $\left(\tau_{\mathrm{p}}^{\mathrm{p}} / \tau>1\right)$ :

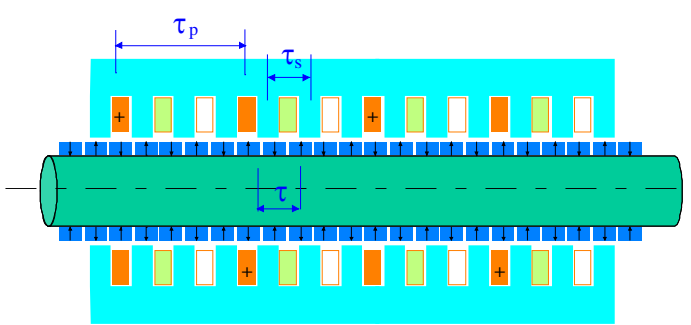

(a)

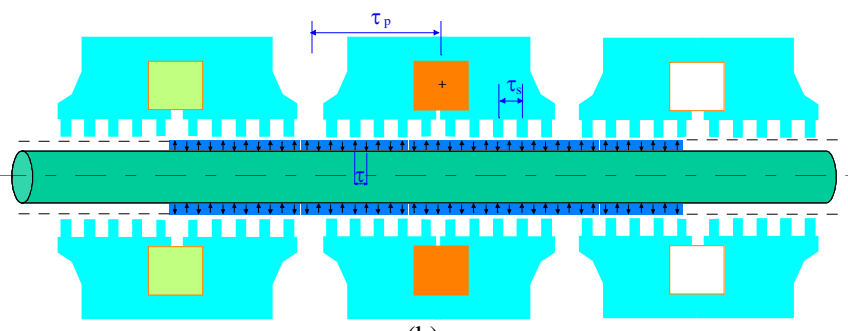

(b)

Fig. 23 Exemples d’actionneurs linéaires tubulaires à couplages fractionnés

La principale modification apportée par ce type de structure est la valeur du rapport $\tau_{\mathrm{p}}^{\mathrm{p}} / \tau$ qui peut être très supérieur à l'unité, ce qui confère à ce type de couplages des performances spécifiques supérieures à celles obtenues par les actionneurs à couplages polaires (voir équations (14) et (15)).
On montre d'autre part que ce découplage des échelles conduit à rendre optimales les dimensions du motif élémentaire (motif de largeur $\tau$ ) essentiellement liées à la valeur du jeu mécanique $\mathrm{e}$ $[16,17]$. On peut ainsi écrire : $\tau_{\mathrm{opt}} \approx \mathrm{k}_{\mathrm{e}} \mathrm{e}$ où $\mathrm{k}_{\mathrm{e}}$ est un coefficient de dépendance qui vaut approximativement 10 sur des considérations de densité d'effort mais cette valeur dégrade le facteur de puissance. Si on souhaite minimiser le coût global, il est nécessaire de trouver des compromis.

Les relations (14) et (15) deviennent :

$$
\begin{aligned}
& \overline{\mathrm{F}} \approx \mathrm{K}_{\mathrm{f}} \cdot\left(\mathrm{A}_{\mathrm{L}} \cdot \delta\right) \frac{\overline{\mathrm{B}_{\mathrm{f} 0}}}{\mathrm{k}_{\mathrm{e}} \cdot \mathrm{e} \cdot \tau_{\mathrm{p}} \ell \mathrm{r}^{\frac{3}{2}}} \\
& \frac{\overline{\mathrm{F}}}{\mathrm{V}_{\mathrm{a}}} \approx \mathrm{K}_{\mathrm{fv}} \cdot\left(\mathrm{A}_{\mathrm{L}} \cdot \delta\right)^{0,5} \frac{\overline{\mathrm{B}_{\mathrm{f} 0}}}{\mathrm{k}_{\mathrm{e}} \cdot \frac{\tau_{\mathrm{p}}}{\sqrt{\mathrm{r}}}}
\end{aligned}
$$

Rappelons que le pas polaire est lié au rayon d'entrefer par les considérations de saturation de la culasse intérieure (voir relation (18)).

Ainsi, si le pas polaire (donc la longueur active $\ell$ ) augmente plus vite que $\sqrt{\mathrm{r}}$, la force volumique se trouve alors améliorée pour des grands volumes. En particulier, si l'on accroît le pas polaire de telle façon que $\tau_{\mathrm{p}} \propto \mathrm{r}$, alors la force et la force volumique augmentent respectivement suivant les lois :

$$
\begin{aligned}
& \overline{\mathrm{F}} \propto\left(\mathrm{r}^{\frac{7}{2}} / \mathrm{e}\right) \\
& \frac{\overline{\mathrm{F}}}{\mathrm{V}_{\mathrm{a}}} \propto(\sqrt{\mathrm{r}} / \mathrm{e}) \propto \frac{1}{\mathrm{e}} \cdot(\overline{\mathrm{F}})^{+\frac{1}{7}} \text { avec } \tau_{\mathrm{p}} \propto \ell \propto \mathrm{r} \propto \mathrm{R}
\end{aligned}
$$

Ce cas est représenté à la figure 24 où l'on constate la supériorité des actionneurs à couplages fractionnés en particulier pour les volumes importants. Pour les volumes très faibles, le rapport $\tau_{\mathrm{p}} / \tau$ tend vers l'unité et donc on retrouve performances des actionneurs à couplages polaires.

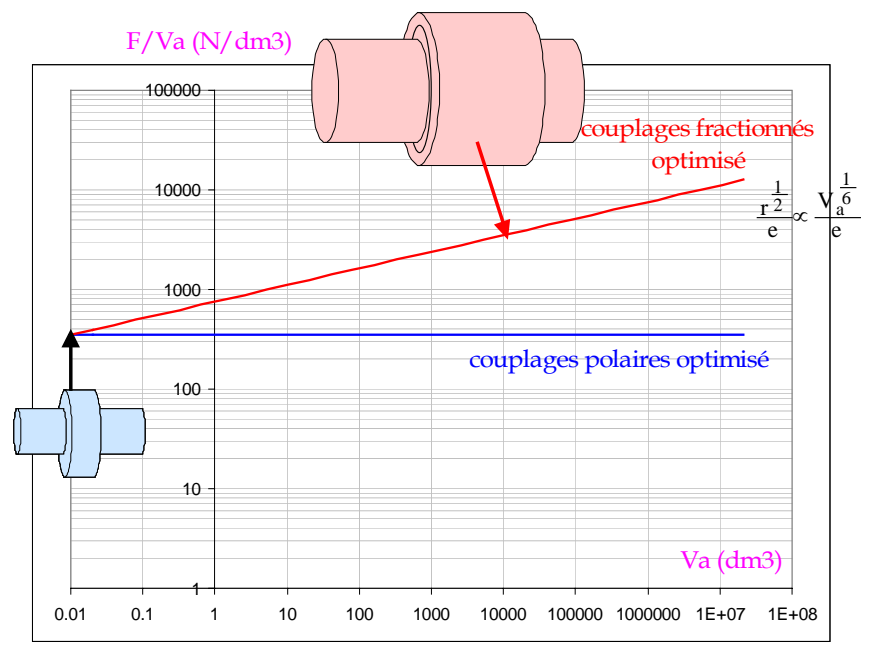

Fig. 24 Effet d'échelle sur la force volumique (avec e conservé)

Remarques :

(1) Le bénéfice en termes de force volumique des actionneurs à couplages fractionnés trouve sa limite, à vitesse de translation imposée, dans l'augmentation de la fréquence de conversion et donc dans l'accroissement des pertes magnétiques et des pertes dues au convertisseur électronique. Ces inconvénients peuvent être atténués par la limitation des vitesses de déplacement (actionneur 
force) ou par l'emploi de matériaux magnétiques « hautes fréquences » tel que les poudres de fer composites.

(2) A l'inverse du couplage polaire, la configuration multi-moteurs appliquée au couplage fractionné défavorise la force volumique. En effet, le maintien des dimensions de l'actionneur et les solutions multi-actionneurs ne font, au mieux, que conserver la force volumique.

\section{ENTRAINEMENTS EM INDIRECTS}

\section{A. Principe}

Diverses solutions mécaniques existent pour la réalisation d'entraînements électromagnétiques indirects. Il s'agit souvent d'accoupler un moteur tournant à la charge en déplacement linéaire par l'intermédiaire d'un système de transformation de mouvement rotation/translation. Parmi les systèmes intermédiaires, on peut citer les systèmes bielle-manivelle, pignon-crémaillière et vis-écrou (le plus employé, figure 7).

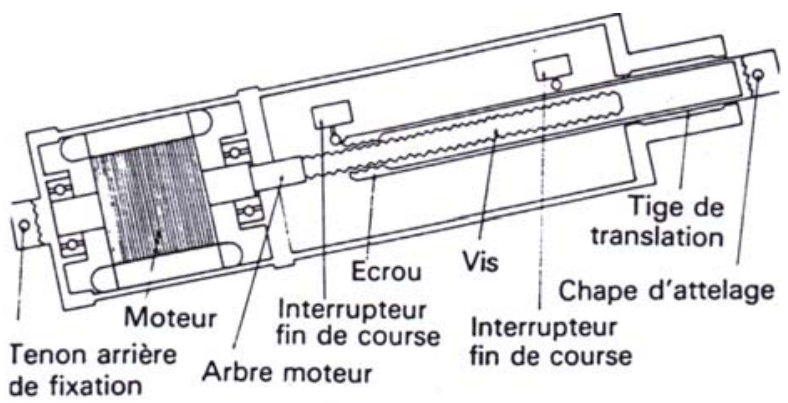

Fig. 25 Schéma de principe d'un entraînement indirect à transmission visécrou

Prenons le cas d'un système vis-écrou et examinons tout d'abord ses performances statiques.

Dans ce cas, l'expression de la force exercée sur la charge linéaire en régime permanent peut s'écrire :

$$
\mathrm{F}=\mathrm{C}_{\mathrm{m}} \cdot \eta_{\mathrm{t}} \mathrm{k}_{\mathrm{t}}
$$

où $\mathrm{C}_{\mathrm{m}}$ est le couple du moteur tournant, $\mathrm{k}_{\mathrm{t}}=\Omega / \mathrm{V}$ est le facteur de transformation proportionnel à l'inverse du pas de vis et $\eta_{t}$ le rendement énergétique de transformation.

Connaissant la loi de variation du couple d'un moteur tournant cylindrique classique (couplage polaire) à induction d'excitation constante (le diamètre du moteur est supposé évolué proportionnellement à la longueur active) [18] :

$$
\mathrm{C}_{\mathrm{m}} \propto(\ell)^{7 / 2}
$$

Nous pouvons en déduire celle de la force volumique (hors volume du système de transformation) :

$$
\mathrm{F} / \mathrm{V}_{\mathrm{a}} \propto \mathrm{F}^{1 / 7} \cdot\left(\mathrm{k}_{\mathrm{t}} \cdot \eta_{\mathrm{t}}\right)^{6 / 7}
$$

Ainsi, la force exercée sur la charge linéaire entraînée ramenée au volume du moteur tournant est d'autant plus importante que le pas de vis est faible ( $\mathrm{k}_{\mathrm{t}}$ élevé).

De plus, le rapprochement des expressions des forces dans les cas d'entraînements indirect et directs (couplages polaires et fractionnés optimisés, relations (19) et (23)) permet de montrer aisément que l'entraînement indirect est d'autant plus intéressant (volume actionneur faible) que les poussée exigées sont importantes (voir figure 26). Cette solution reste néanmoins moins performante comparativement à la solution direct à couplage fractionné compte tenu de l'accroissement du volume global généré par le système de transformation de mouvement non pris en compte ici. Ce volume supplémentaire est notamment proportionnel à la course du mover.

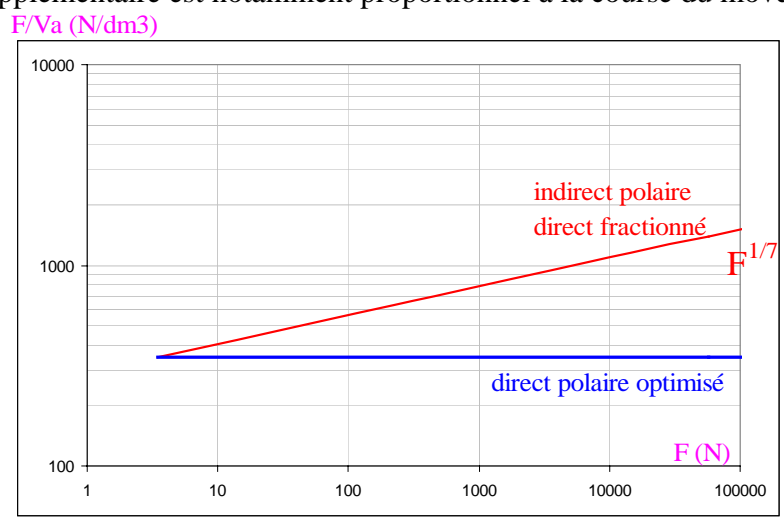

Fig. 26 Effets d'échelle sur l'effort volumique : comparaison des entraînements directs et indirect (volume du système de transformation de mouvement non pris en compte)

Concernant les performances dynamiques, celles-ci sont comparées simplement à partir des capacités d'accélération des différentes solutions.

Reprenons le cas d'entraînement indirect vis-écrou. L’équation mécanique côté moteur tournant s'écrit :

$$
\left.C_{m}=\gamma\left[J_{m}\left(\frac{\Omega}{v}\right)+\frac{M}{\eta_{t}(\Omega / v}\right)\right]
$$

où $\mathrm{M}$ est la masse à déplacer, $\Omega$ et $\mathrm{v}$ les vitesses de translation et de rotation respectivement, $\mathrm{J}_{\mathrm{m}}$ est l'inertie du moteur tournant et $\gamma=\frac{\mathrm{dV}}{\mathrm{dt}}$ est l'accélération de la charge en $\mathrm{m} / \mathrm{s}^{2}$.

Sur la figure ci-dessous, sont représentées l'accélération à couple donné et le couple à accélération donnée en fonction du facteur $k_{t}$ où sont indiquées également les expressions de l'accélération maximale $\gamma_{\max }$, du couple minimal $C_{\min }$ et du facteur de transformation optimal $\mathrm{k}_{\mathrm{t} \_o p t}$.

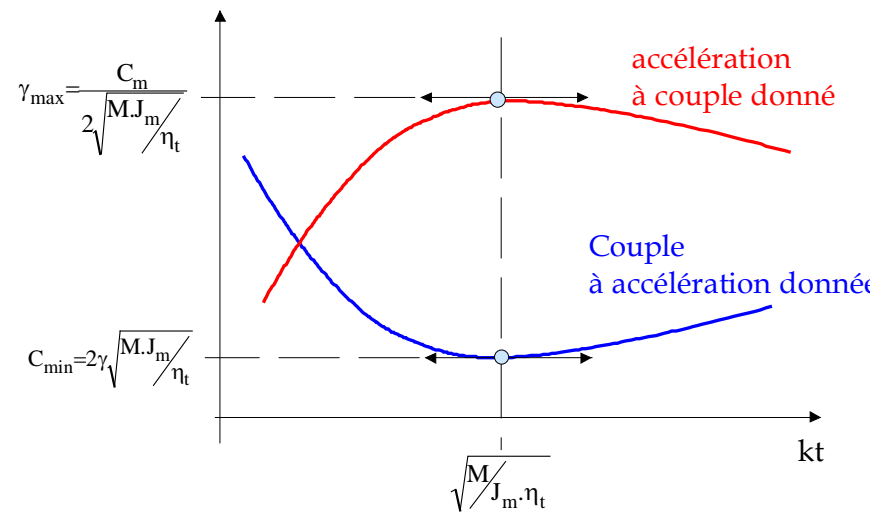

Fig. 27 Couple et accélération en fonction du facteur de transformation $\mathrm{k}_{\mathrm{t}}$

Sachant que $\mathrm{J}_{\mathrm{m}} \propto(\ell)^{5}$ et connaissant la loi de variation du couple de la machine tournante (relation (25)), l'accélération maximale de la charge linéaire varie alors suivant la loi :

$$
\gamma_{\max } \propto \ell \cdot M^{-0,5}
$$

Le volume actif du moteur tournant (toujours hors système de transformation) ainsi que le facteur optimal de transformation varient donc en fonction de l'accélération imposée et de la masse entraînée suivant la loi : 
$\mathrm{V}_{\mathrm{a}} \propto\left(\gamma_{\max }\right)^{3} \cdot \mathrm{M}^{+\frac{3}{2}}$

$\mathrm{k}_{\mathrm{t} \_\mathrm{opt}} \propto\left(\gamma_{\text {max }}\right)^{-\frac{5}{2}} \cdot \mathrm{M}^{-\frac{3}{4}}$

Pour les entraînements directs (sans intermédiaire), nous avons :

$\mathrm{V}_{\mathrm{a}}=\gamma_{\text {max }} \cdot \mathrm{M} \quad$ : couplages polaires

$\mathrm{V}_{\mathrm{a}}=\left(\gamma_{\max }\right)^{\frac{6}{7}} \cdot \mathrm{M}^{\frac{6}{7}}$ : couplages fractionnés

A tire d'exemple, la figure 28 présente l'évolution du volume actif de l'actionneur en fonction de l'accélération souhaitée pour une masse entraînée de 10 kg.

Ainsi, ce résultat bien que qualitatif, indique clairement la supériorité en termes d'encombrement et d'accélération, des systèmes à entraînement direct en particulier le système utilisant le couplage fractionné.

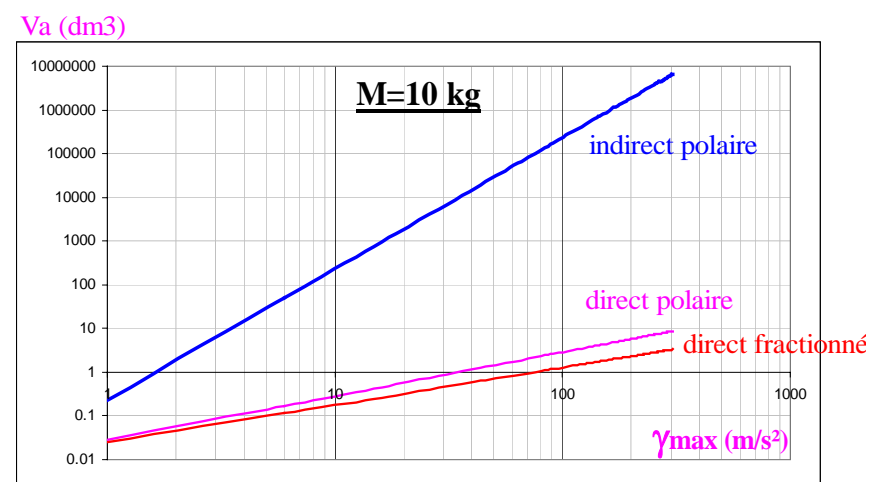

Fig. 28 Volume actionneur en fonction de l'accélération maximale pour $\mathrm{M}=10 \mathrm{~kg}$ (volume système de transformation non pris en compte et $\mathrm{k}_{\mathrm{t}}=\mathrm{k}_{\mathrm{topt}}$ )

\section{ENTRAINEMENTS ELECTRO-HYDRAULIQUES}

\section{A. Principe}

Le principe général d'un entraînement électro-hydraulique (valable également dans une certaine mesure aux entraînements pneumatiques) est la génération d'une poussée sur un piston à travers la mise sous pression d'un liquide dans une chambre. La figure ci-dessous en présente les principaux constituants dans le cas d’un vérin classique double effet [19].

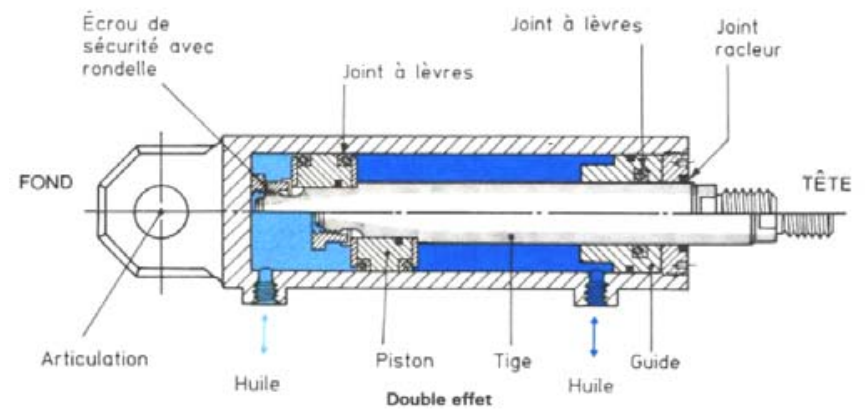

Fig. 29 Schéma général d'un vérin hydraulique classique double effet [19]

Dans le cas d'un actionneur hydraulique à puissance électrique, l'alimentation en huile de l'actionneur est réalisé généralement à partir d'éléments supplémentaires que sont (voir figure 30), le réservoir, la pompe, un réducteur de vitesse éventuel et un moteur électrique tournant.

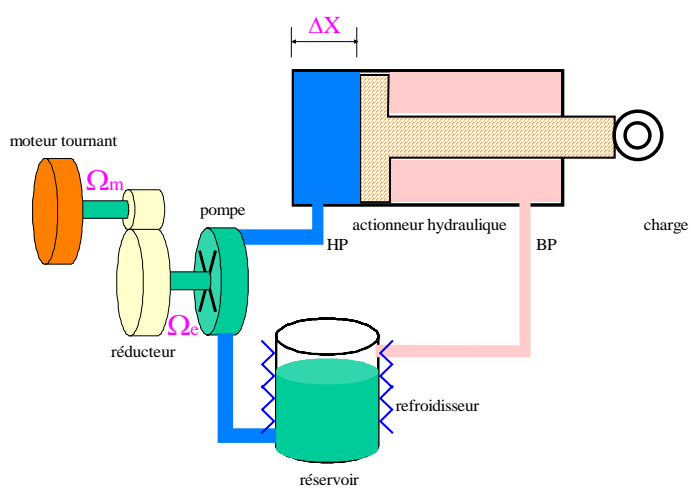

Fig. 30 Schéma de principe d'un entraînement hydraulique à puissance électrique

Pour des courses et des vitesses de déplacements faibles, le système d'entraînement électro-hydraulique peut être extrêmement compact. Un exemple est donné à la figure 31 représentant un actionneur dit de type EHA pour l'actionnement d'aileron (Airbus A320) [25].

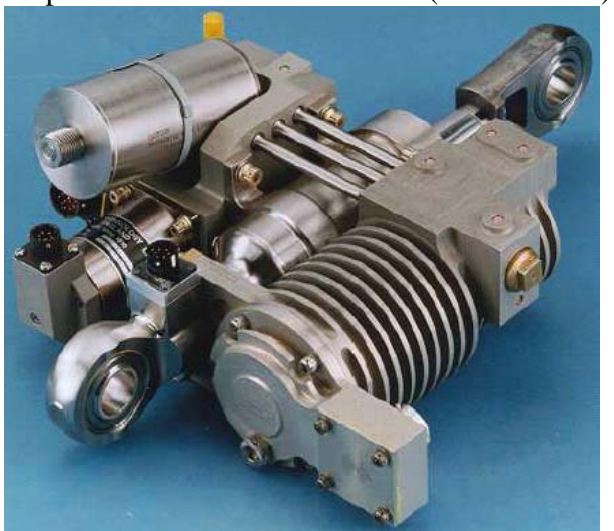

Fig. 31 Photo d'un actionneur de type EHA (Airbus A320 Aileron) [25]

\section{B. Performances}

La force théorique générée dans ce type de système est directement liée à la pression $\sigma_{\mathrm{n}}$ du liquide dans la chambre de compression (en $\mathrm{Pa}$, avec $1 \mathrm{~Pa}=10^{-5}$ bars $=10^{-4} \mathrm{~N} / \mathrm{cm}^{2}$ ) et à la section de la tige (pour un simple effet) ou du piston (pour un double effet) :

$$
\mathrm{F}=\sigma_{\mathrm{n}} \cdot \mathrm{S}
$$

Les valeurs courantes de pression rencontrées sont de l'ordre de 100 bars $\left(1000 \mathrm{daN} / \mathrm{cm}^{2}\right)$, ce qui donne pour un diamètre d'alésage de $100 \mathrm{~mm}$, une poussée de l'ordre de 785 daN. Notons que pour les actionneurs pneumatiques, les pressions atteintes sont nettement moins importantes (quelques bars).

Ainsi, si l'on considère la pression réalisable limitée (100 à 300 bars), la force de l'actionneur impose directement la section du piston.

La force développée ramenée au volume de la chambre de compression s'écrit :

$\mathrm{F} / \mathrm{V}_{\mathrm{a}}=\frac{\sigma_{\mathrm{n}}}{\Delta \mathrm{X}}$ où $\mathrm{V}_{\mathrm{a}}=\mathrm{S} . \Delta \mathrm{X}$

Comme l'illustre la figure 32 correspond à une pression limite de 100 bars, si l'on fait abstraction des éléments supplémentaires signalés précédemment (ceux-ci prennent un volume non négligeable dans le cas des déplacements de grandes courses), les entraînements hydrauliques présentent des forces volumiques et massiques nettement supérieures aux systèmes électromagnétiques pourvu que la course soit très faibles (quelques $\mathrm{cm}$ ). La force 
volumique des systèmes EM multi-pas est à l'inverse indépendante de la course.

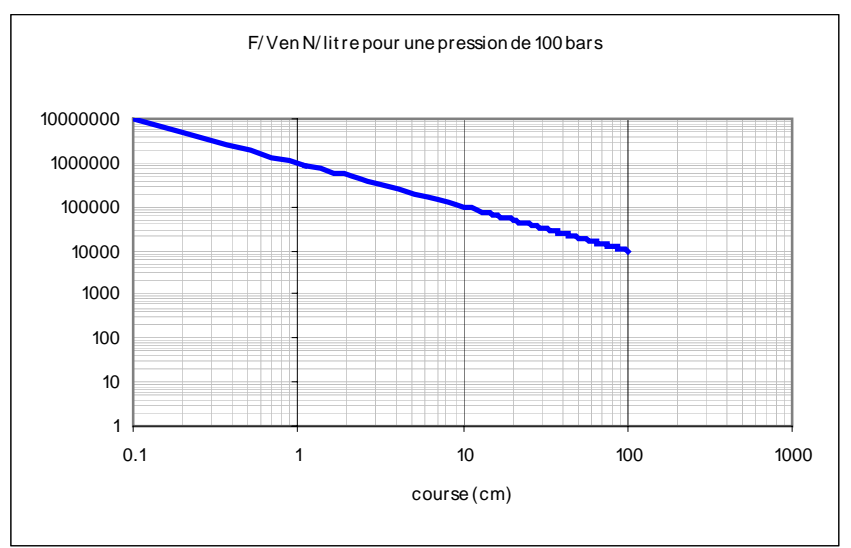

Fig. 32 Force volumique (N/dm $\left.{ }^{3}\right)$ en fonction de la course (cm) d'un entraînement hydraulique (pression maximale = 100 bars)

La vitesse de déplacement v est quant à elle liée au débit d’huile Q (en $\left.\mathrm{m}^{3} / \mathrm{s}\right)$ et à la section du piston $\mathrm{S}$ :

$\mathrm{V}=\frac{\mathrm{Q}}{\mathrm{S}}$

Le débit souhaité conditionne directement la cylindrée $V_{p}$ de la pompe ainsi que la section des canalisations hydrauliques. Dans le cas d'une pompe volumétrique, nous avons la relation :

$\mathrm{Q}=\mathrm{V}_{\mathrm{p}} \cdot \Omega_{\mathrm{p}}$

Les débits usuels sont de l'ordre de 1000 l/min obtenus pour des vitesses de rotation pompe allant de $100 \mathrm{tr} / \mathrm{min}$ (pompes lentes) à $10000 \mathrm{tr} / \mathrm{min}$ (pompes rapides) [26].

Précisons que le volume du réservoir (voir figure 30) est directement lié au débit par la relation empirique suivante :

$\mathrm{V}_{\text {rés }}=2 . \mathrm{Q} . \mathrm{t}_{0} \quad$ où $\mathrm{t}_{0} \approx 1 \mathrm{~min}$

Par conséquent, si l'on impose des valeurs limites de pression et de débit, la puissance de l'actionneur hydraulique est imposée, celle de la pompe et du moteur d'entraînement le sont également, au rendement de conversion près. La vitesse de déplacement varie donc suivant la loi :

$$
\mathrm{v}=\frac{\mathrm{Q} \cdot \sigma_{\mathrm{n}}}{\mathrm{F}}
$$

Notons que cette relation ne tient pas compte d'autres limitations liée notamment aux pertes "mécaniques" dues au cisaillement huile/piston.

Compte tenue de ces différentes limites et dans le cas de très fortes poussées, les vitesses de déplacements classiquement obtenues sont relativement faibles, de l'ordre de $0.2 \mathrm{~m} / \mathrm{s}$ à $5 \mathrm{~m} / \mathrm{s}$.

\section{CONCLUSION}

Après un rappel des topologies existantes d'actionneurs linéaires, nous avons tenté dans cette étude de montrer et d'analyser les évolutions des performances limites des actionneurs à aimants permanents en termes de performances volumiques et d'accélération maximale.

Nous avons ainsi montré que les actionneurs directs à couplages fractionné (Vernier ou global) permettent des densités volumiques de force importantes, proportionnelles au volume de l'actionneur.
Alors que celles obtenues par les structures à couplages polaires (classiques) sont limitées à $400 \mathrm{~N} / \mathrm{dm}^{3}$ dans des conditions de fonctionnement usuelles. D'autre part, les configurations polyentrefers permettent d'améliorer encore sensiblement ces performances [21,23].

La disposition d’un système mécanique intermédiaire de transformation de mouvement (entraînements indirects) permet d'accroître notablement la force volumique des actionneurs à couplage polaire (classique) (hors système de transformation). Au prix cependant d'une complexité accrue de la chaîne de traction et d’une limitation, à volume comparé, des accélérations de la charge.

Outre ces avantages, les systèmes électrique à entraînement direct ont de plus :

- une plus grande précision du positionnement

- une meilleure fiabilité et durée de vie accrue

- une plus grande flexibilité d'utilisation (partie indépendante sur un même axe)

Enfin, les actionneurs électro-hydrauliques offrent des possibilités de poussées spécifiques très supérieures aux solutions électromagnétiques, essentiellement pour des courses et des vitesses de déplacement faibles. Dans ces conditions, l'intégration moteur/pompe/vérin permet de concevoir des actionneurs force extrêmement compacts.

\section{REFERENCES}

[1] Jacek F. Gieras "Status of linear Motors in the United Sates », $4^{\text {th }}$ Int. Symp. on Linear Drives for Industry Application (LDIA), September 2003, Birmingham (UK).

[2] A. Cassat, N. Corsi, R. Moser, N. Wavre « Direct linear drives : Market and Performance Status », $4^{\text {th }}$ Int. Symp. on Linear Drives for Industry Application (LDIA), Sep. 2003, Birmingham (UK).

[3] Hörmann O., «Linear Direct Drives: Dynamic, Economics, Innovative », Intelligent Motion, June 1993 proceedings. pp. : 82-87.

[4] Teruo Azukizawa «Status of linear drives in Japan », $4^{\text {th }}$ Int. Symp. on Linear Drives for Industry Application (LDIA), September 2003, Birmingham (UK).

[5] Sagansan A., Boyer S. «Les actionneurs électriques et l'aéronautique », Colloque CEMD, ENS Cachan, 4 février 1999, pp : 135-141.

[6] B. Nogarede « Machines tournantes : conversion électromécanique de l'énergie », Techniques de l’Ingénieur, D 3410.

[7] Staton D.A., Deodhar R.P., Soong W.L., Miller T.J.E. «Torque prediction using the flux-mmf diagram in AC, DC and reluctance motors ». Proceedings IEEE trans. on Ind. Appl., vol. 32, Jan./Feb. 1996, pp. : 180-188.

[8] P.E. Cavarec, H. Ben Ahmed, B. Multon, M. Antunes « Actionneurs électromagnétiques: classification topologiques », Techniques de l’Ingénieur, D3 412 à paraître.

[9] B. Nogarede " Machines tournantes: principes et constitution ", Techniques de l’Ingénieur, D 3411.

[10] Matt. D., Llibre J.F. « Performances comparées des machines à aimants et à réluctance variable. Maximisation du couple massique ou volumique », JPIII, octobre 1995, № 5, pp : 1621-1641.

[11] P.E. Cavarec « Conception, modélisation et optimisation des actionneurs polyentrefers à bobinages globaux: application aux structures linéaires multi-tiges ", thèse de doctorat de l'ENS Cachan (SATIE), nov. 2002 (téléchargeable sur le site de l'ENS-bretagne).

[12] A. dos santos " Transverse flux linear motor leading to a tubular configuration ", $4^{\text {th }}$ Int. Symp. on Linear Drives for Industry Application (LDIA), September 2003, Birmingham (UK).

[13] Hiroyuki Ohsaki « Electromagnetic characteristics of a coreless surface motor using Halbach permanent magnet ", $4^{\text {th }}$ Int. Symp. on Linear Drives for Industry Application (LDIA), September 2003, Birmingham (UK).

[14] Ben Ahmed H., Multon B., Prévond L., Lucidarme J. « Actionneur linéaire intégré pour application domotique », Revue 3EI, $N^{\circ} 21$, juin 2000. 
[15] Jack A.G et. al.. « Permanent-Magnet Machines with Powder Iron Cores and Prepressed Windings », IEEE Trans. On Industry app., vol. 36, N4, july/august 2000, pp.:1077-1084.

[16] P.E. Cavarec, H. Ben Ahmed, B. Multon « Force density improvements from increasing ther number of airgap surfaces in synchronous linear actuators », Revue IEE proc. Elec. Power Appl., vol. 150, N 1, January 2003, pp.:106-116.

[17] Ben Ahmed H., Desesquelles P.F., Lucidarme J., « Méthode seminumérique de pré-dimensionnement des machines à aimants permanents et à bobinage global ", Journal de Physique III, october 1995, pp : 703-725.

[18] Multon B., J. Bonal « Entraînements électromécaniques directs : diversité, contraintes et solutions », Colloque CEMD, ENS Cachan, 4 février 1999, pp : 1-14.

[19] Affouard R. « Vérins hydrauliques », Techniques de l’Ingénieur, B6 040 .

[20] Favre E., Brunner C. Piaget D. « Actionneurs linéaires directs », Colloque CEMD, ENS Cachan, 4 février 1999, pp.:31-44.

[21] Lucidarme J., Amouri A., Poloujadoff M., «Optimum design of longitudinal field variable reluctance motors», IEEE trans. on Energy Conversion, vol. 8, n³, September 1993, pp. : 357-361.

[22] H BenAhmed, L Prevond, B Multon, B Salamand, J Lucidarme, «Special synchronous linear actuators : Structures and performances», Electromotion n5 p93 à 101, 1998

[23] H. Ben Ahmed , L. Prévond, B. Multon «Structures polyentrefers : solutions pour les entraînements directs ? », Colloque CEMD, ENS Cachan, 4 février 1999, pp : 95-100.

[24] H.Ben Ahmed, P.E.Cavarec, L.Prévond, B.Multon «Conception et étude d'un actionneur linéaire synchrone à aimants permanents et multi-plaques », Electrotechnique du Futur 14-15 novembre 2001 Nancy pp.307-312.

[25] P. W. Wheeler et al. «A matrix converter Motor Drive for an Aircraft actuation System », EPE 2003, Toulouse.

[26] S. Calloch, J.Y. Cognard, D.Dureisseix, D. Marquis « Les systèmes de transmission de puissance», édition Hermes, 2003.

[27] Site internet Avcon Inc. (US) http://www.calinear.com/

[28] Site internet Etel S.A. (CH) ML 2020 : http://www.etel.ch/

[29] Site internet Trilogy Systems : http://www.trilogysystems.com/

[30] Site internet Aerotech : $\underline{\text { http://www.aerotech.com/ }}$

Site des constructeurs et vendeurs européens d'actionneurs de tous types : http://www.trem.biz/fr/produits02/actionneurs_fournisseurs.htm

Site de l'ENS-Antenne de Bretagne : http://www.bretagne.ens-cachan.fr Site SATIE : http://www.satie.ens-cachan.fr

\section{LISTE DES PRINCIPALES NOTATIONS}

\begin{tabular}{|c|c|}
\hline $\mathrm{A}_{\mathrm{L}}$ & : densité linéique efficace de courant d’induit (A/m) \\
\hline$\alpha$ & : coefficient d'échange thermique $\left(\mathrm{W} \cdot \mathrm{m}^{-2} \cdot \mathrm{K}^{-1}\right)$ \\
\hline $\mathrm{B}_{\text {sat }}$ & : induction au coude de saturation du matériau magnétique \\
\hline$\overline{\mathrm{B}_{\mathrm{f} 0}}$ & : induction inducteur moyenne pour $\mathrm{x}$ tel que $\mathrm{i}(\mathrm{x})=0(\mathrm{~T})$ \\
\hline $\mathrm{B}_{\mathrm{r}}$ & : induction rémanente des aimants $(\mathrm{T})$ \\
\hline $\mathrm{B}_{\mathrm{m}}$ & : induction maximale dans la culasse ou dans les dents $(\mathrm{T})$ \\
\hline $\mathrm{C}_{\mathrm{m}}, \mathrm{C}_{\mathrm{ch}}$ & : couple du moteur tournant et couple de charge \\
\hline$\delta$ & : densité efficace de courant d'induit (A/m²) \\
\hline$\Delta \theta$ & : élévation de température (K) \\
\hline$\gamma$ & : accélération linéaire $\left(\mathrm{m} / \mathrm{s}^{2}\right)$ \\
\hline $\mathrm{h}_{\mathrm{a}}$ & : hauteur des aimants (m) \\
\hline $\mathrm{h}_{\mathrm{b}}$ & : hauteur de la fenêtre bobinable (m) \\
\hline$h=\frac{h_{b}}{r}$ & : hauteur relative de la fenêtre bobinable \\
\hline $\mathrm{e}$ & : entrefer $(m)$ \\
\hline $\mathrm{e}_{\mathrm{c}}$ & : épaisseur culasse (m) \\
\hline$\eta_{t}$ & : rendement du système de transformation de mouvement \\
\hline
\end{tabular}

$\mathrm{I}, \mathrm{I}_{\max }$ : courants efficace et maximal d'induit (A)

$\mathrm{J}_{\mathrm{m}} \quad$ : inertie du moteur tournant (kg.m²)

$\mathrm{k}_{\mathrm{r}} \quad$ : coefficient de remplissage de cuivre

$\mathrm{k}_{\mathrm{Cf}} \quad$ : facteur de remplissage axial cuivre/fer

$\mathrm{K}_{\mathrm{f}}, \mathrm{K}_{\mathrm{fv}}$ : coefficients d'effort et d'effort volumique

$\mathrm{k}_{\mathrm{e}}=\tau / \mathrm{e} \quad$ : facteur (rapport pas de conversion/entrefer)

$\mathrm{k}_{\mathrm{t}} \quad$ : facteur de transformation de mouvement

$\mathrm{k}_{\text {imoy }}, \mathrm{k}_{\text {ieff }}$ : facteurs de forme du courant d'induit

$\mathrm{k}_{\mathrm{gr}}=\mathrm{k}_{\mathrm{r}} \cdot \mathrm{k}_{\mathrm{cf}}$ : coefficient global de remplissage cuivre

$\ell \quad$ : longueur active de l'actionneur (m)

$\ell_{\mathrm{b}} \quad$ : longueur moyenne d'une spire du bobinage d'induit (m)

M : masse (kg)

$\mathrm{n}, \mathrm{N}_{\mathrm{b}} \quad$ : nombre de spires par bobine et nombre total de bobines

$R(x) \quad$ : fonction unitaire de répartition du flux inducteur

r,R : rayon d'entrefer et rayon extérieur (m)

$\rho \quad$ : résistivité du conducteur $(\Omega . \mathrm{m})$

$S_{\mathrm{p}} \quad$ : section d'un pôle d'induit $\left(\mathrm{m}^{2}\right)$

$\mathrm{S}_{\mathrm{th}} \quad$ : surface d'échange thermique $\left(\mathrm{m}^{2}\right)$

$\tau_{\mathrm{p}} \quad$ : pas polaire d'induit (m)

$\tau \quad$ : pas de conversion (m)

$\mathrm{V} \quad$ : vitesse de translation du mover et/ou de la charge

$\mathrm{V}_{\mathrm{a}} \quad$ : volume actif de l'actionneur $\left(\mathrm{m}^{3}\right)$

$\mathrm{v}_{\mathrm{cu}} \quad$ : volume cuivre $\left(\mathrm{m}^{3}\right)$

$\mathrm{X} \quad$ : position de la partie mobile ou du mover $(\mathrm{m})$

X : course du mover (m)

Remerciement: Les auteurs remercient, pour son aide et ses précieux éclaircissements, M. C. Kerzhého, professeur agrégé de mécanique à l'ENS Cachan - Antenne de Bretagne. 